\title{
Kidney-infiltrating T cells in murine lupus nephritis are metabolically and functionally exhausted
}

\author{
Jeremy S. Tilstra, ${ }^{1,2}$ Lyndsay Avery, ${ }^{1,3}$ Ashley V. Menk, ${ }^{4}$ Rachael A. Gordon, ${ }^{1}$ Shuchi Smita, ${ }^{1}$ Lawrence P. Kane, ${ }^{1,5}$ Maria Chikina, ${ }^{6}$ \\ Greg M. Delgoffe, ${ }^{1,4,5}$ and Mark J. Shlomchik ${ }^{1}$ \\ ${ }^{1}$ Department of Immunology, ${ }^{2}$ Department of Medicine, ${ }^{3}$ Infectious Disease and Microbiology Graduate Program, ${ }^{4}$ Tumor Microenvironment Center, ${ }^{5}$ Cancer Immunology Program, and ${ }^{6}$ Department of \\ Computational and Systems Biology, University of Pittsburgh School of Medicine, Pittsburgh, Pennsylvania, USA
}

\begin{abstract}
While T cells are important for the pathogenesis of systemic lupus erythematosus (SLE) and lupus nephritis, little is known about how T cells function after infiltrating the kidney. The current paradigm suggests that kidney-infiltrating T cells (KITs) are activated effector cells contributing to tissue damage and ultimately organ failure. Herein, we demonstrate that the majority of CD4+ and CD8+ KITs in 3 murine lupus models are not effector cells, as hypothesized, but rather express multiple inhibitory receptors and are highly dysfunctional, with reduced cytokine production and proliferative capacity. In other systems, this hypofunctional profile is linked directly to metabolic and specifically mitochondrial dysfunction, which we also observed in KITs. The T cell phenotype was driven by the expression of an "exhausted" transcriptional signature. Our data thus reveal that the tissue parenchyma has the capability of suppressing $T$ cell responses and limiting damage to self. These findings suggest avenues for the treatment of autoimmunity based on selectively exploiting the exhausted phenotype of tissue-infiltrating T cells.
\end{abstract}

\section{Introduction}

Systemic lupus erythematosus (SLE) is a systemic autoimmune disease defined by loss of self-tolerance, autoantibody formation, cellular-tissue infiltration, and end-organ damage. The most common and severe organ-specific manifestation of SLE is lupus nephritis (LN), with $10 \%$ of patients developing end-stage renal disease (1). There are no FDA-approved therapies for LN, and current treatments are nonspecific, with suboptimal remission rates (1-3). The importance of $\mathrm{T}$ cells in SLE has been well documented in murine models, with a consensus that $\mathrm{CD} 4^{+} \mathrm{T}$ cells propagate the disease (4-7). The role of $\mathrm{CD}^{+} \mathrm{T}$ cells in disease pathogenesis is less clear. Several studies have shown that MHC class I is required $(8,9)$, while others have concluded that disease pathogenesis does not require $\mathrm{CD}^{+} \mathrm{T}$ cells $(5,6)$. Recently the immunosuppressive drug tacrolimus, which targets $\mathrm{T}$ cells, has been shown to be efficacious in the treatment of LN (10), further supporting the importance of T cells in LN pathogenesis.

While the nature of kidney-infiltrating cells has been investigated, a much greater focus in murine and human lupus studies has been on the more readily accessible peripheral cell populations, which are relatively well understood. Comparatively much less is known about organ-specific disease processes, even though infiltrating cells directly affect target tissues and hence should be a priority to understand.

$\mathrm{T}$ cells make up the majority of kidney-infiltrating cells in humans (11-13) and murine models of lupus (14). A limited number

Conflict of interest: The authors have declared that no conflict of interest exists. License: Copyright 2018, American Society for Clinical Investigation.

Submitted: March 2, 2018; Accepted: August 8, 2018.

Reference information: J Clin Invest. 2018;128(11):4884-4897.

https://doi.org/10.1172/JCl120859. of prior studies evaluating the role of kidney-infiltrating $\mathrm{T}$ cells (KITs) in LN have shown that they undergo clonal expansion (15, 16) and are $\mathrm{CD}_{4} 4^{+}(17)$, suggesting an activated phenotype. KITs from LN samples evaluated using microdissection showed expression of IL-2, IL-10, IL-17, IL-4, IL-13, and IFN- $\gamma$ transcripts (18). IL-17 protein has been detected by immunofluorescence staining in KITs (19); however, genetic deletion of IL-17 did not affect disease in murine models (20).

Based on these studies, the prevailing hypothesis in the field is that KITs are likely to be activated effector T cells (21). This hypothesis has yet to be tested, as the function and metabolic capacity of KITs have not been directly examined. The metabolic status of KITs is of particular interest, given recent work showing that altering metabolic signaling ameliorates disease (22). Here, to address this gap and determine whether T cells in target tissues differentiate into highly activated and functional cells, as thought, we directly examined the phenotype, metabolism, and functional capacity of KITs in murine lupus models and compared these cells with their peripheral $\mathrm{T}$ cell counterparts.

\section{Results}

Three murine models of LN were used to determine the role and phenotype of KITs. The first model was the MRL.Fas ${ }^{l p r}$ (MRL/lpr) model of lupus, which recapitulates nearly all features of human disease and is therefore a predominant research model (23-28). Importantly, preclinical therapeutic trials in the MRL/lpr model have accurately predicted responses in human translational studies (29-33). The second model was the $\mathrm{Fcgr}_{2} \mathrm{~b}^{-/}$.Y-chromosomelinked autoimmune accelerator $\left(F c g r 2 b^{-1-} . \mathrm{Yaa}\right)$ model, in which deletion of the inhibitory receptor (IR) Fc $\gamma$ RIIB combined with the Yaa mutation results in a proliferative glomerulonephritis in male mice (34). We conducted confirmatory experiments in a third murine model, the MRL.Tlr $9^{-/-}$model, which is sufficient for Fas, 
A

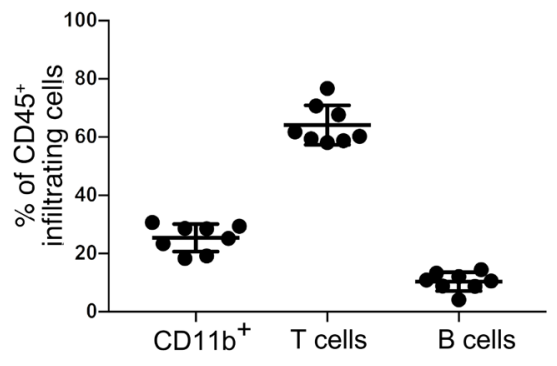

B

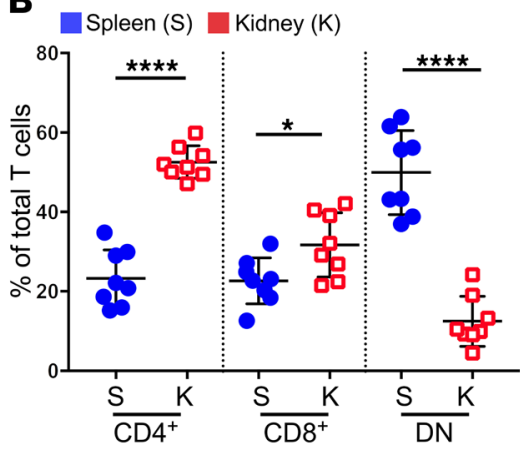

C

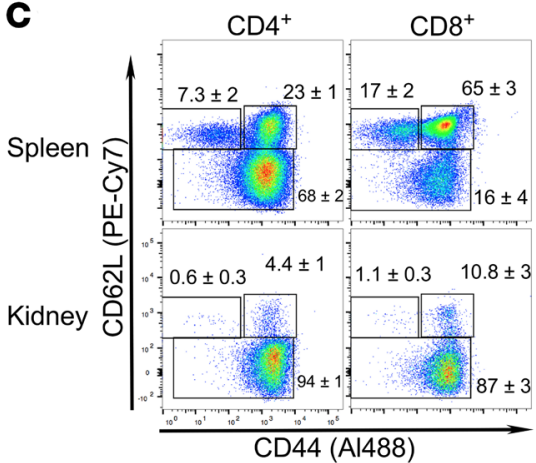

D
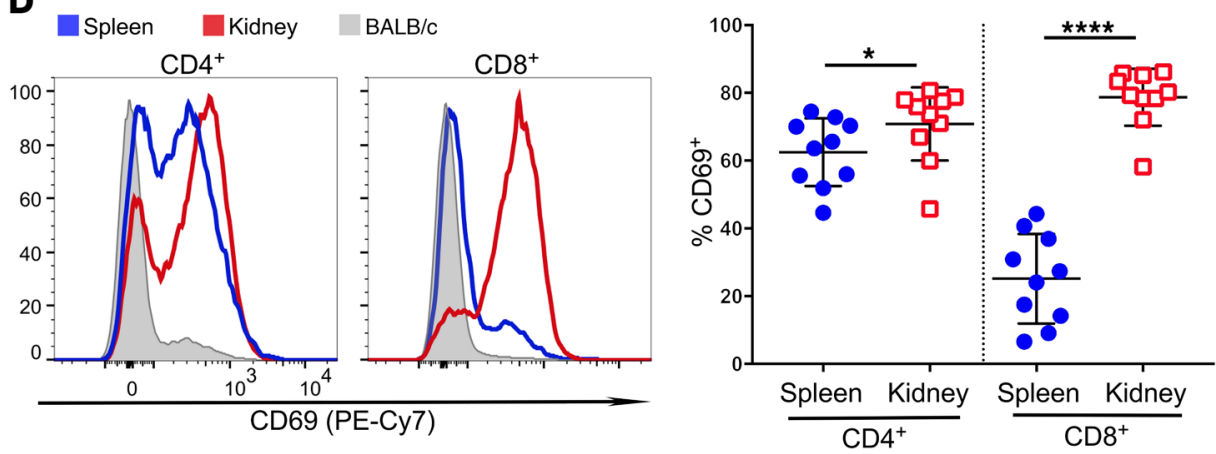

Figure 1. Characterization of $T$ cell populations infiltrating the kidneys of nephritic MRL/lpr mice. (A) The frequency of CD45 ${ }^{+} C D 11 b^{+}$cells, $T$ cells (CD45 ${ }^{+} \mathrm{TCR}^{+}$), and B cells (intracellular Ig $\kappa^{+}$) in kidney infiltrates was determined using flow cytometry ( $n=8$ per group). (B) The frequency of different T cell subsets was determined in the kidneys and spleens of matched MRL/Ipr mice with nephritis ( $n=8$ per group). DN, double negative. (C) Representative flow plots of CD44 and CD62L expression on CD4+ and CD8 ${ }^{+} T$ cells from indicated organs are shown with percentages \pm SD of each gated subpopulation,

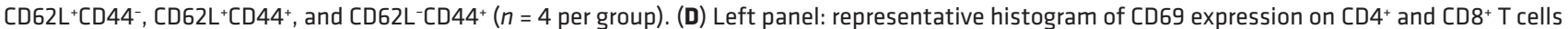
from indicated organs (blue, spleen; red, kidney; gray, BALB/c). Right panel: summary data from spleens and kidneys of lupus-prone mice ( $n=10$ mice per group). For tabulated data, each dot denotes an individual mouse, horizontal lines represent the mean, and error bars show \pm 1 SD. Paired Student's $t$ test was used to determine statistical significance between spleen and kidney samples. ${ }^{*} P<0.05$; ${ }^{* * *} P<0.0001$.

but uses TLR9 deficiency as a disease accelerator. As nonautoimmune mice have very few T cells in their kidneys, the paradigm we have used in this work is to compare KITs in diseased kidneys with $\mathrm{T}$ cells in the spleens of the same mice. However, to determine whether an inflammatory environment was necessary to alter $\mathrm{T}$ cell phenotype, we also examined KITs from C57BL/6 (B6) mice.

$T$ cells make up the majority of kidney-infiltrating immune cells in lupus-prone mice. In aged nephritic MRL/lpr mice, T cells made up $64.2 \%$ of the total $\mathrm{CD} 45^{+}$kidney infiltrate, followed by $\mathrm{CD}_{11 \mathrm{~b}}{ }^{+}$ populations, which accounted for $25.4 \%$ (Figure $1 \mathrm{~A}$ ). We recovered an average of nearly $4 \times 10^{6}$ total TCR- $\beta^{+}$cells from the kidneys of older $\mathrm{MRL} / \mathrm{lpr}$ mice and approximately 10-fold fewer cells in youner, 11- to 12-week-old MRL/lpr mice (Supplemental Figure 1A; supplemental material available online with this article; https://doi.org/10.1172/ JCI120859DS1). The overall composition of $\mathrm{T}$ cell populations in the kidney differed from that in the periphery, with an increased percentage of $\mathrm{CD}^{+}$and $\mathrm{CD}^{+}$and a lower frequency of doublenegative (DN) T cells in the kidney (Figure 1B). Consistent with previous reports (17), KITs expressed elevated amounts of activation markers CD44 and CD69 (Figure 1, C and D) and reduced amounts of CD62L (Figure 1C), suggesting a change in migratory status.

In comparison, there were nearly 100 -fold fewer TCR- $\beta^{+}$cells isolated from the kidneys of B6 mice compared with MRL/lpr mice of similar ages (Supplemental Figure 1A). T cells made up approximately $10 \%$ and $C D 11 b^{+}$cells accounted for $80 \%$ of nonparenchy- mal cells isolated (Supplemental Figure 1B). Kidney-derived T cells from $\mathrm{B} 6$ mice exhibited an increased frequency of $\mathrm{T}$ cells with an effector phenotype $\left(\mathrm{CD} 44^{\mathrm{hi}} \mathrm{CD} 62 \mathrm{~L}^{\mathrm{lo}}\right)$ and increased frequency of $\mathrm{CD} 69^{+}$cells when compared with splenic T cells. Compared with $\mathrm{MRL} / \mathrm{lpr}$ KITs, B6 KITs had an increased frequency of naive $\mathrm{CD} 8^{+}$ $\mathrm{T}$ cells $\left(\mathrm{CD} 44^{\text {lo }} \mathrm{CD} 62 \mathrm{~L}^{\text {hi }}\right)$ and a lower frequency of $\mathrm{CD} 69^{+} \mathrm{CD} 4^{+}$ and $\mathrm{CD} 8^{+} \mathrm{T}$ cells (Supplemental Figure 1, C and D).

KITs are functionally suppressed compared with splenic-derived $T$ cells. Because $\mathrm{T}$ cell infiltrates have a known role in pathogenesis of SLE in murine models and are the predominant cell in the inflamed kidney, we next evaluated the functional status of KITs. Compared with splenic $\mathrm{T}$ cells, a significantly smaller percentage of $\mathrm{CD} 4^{+}$and $\mathrm{CD}^{+} \mathrm{KITs}$ produced inflammatory cytokines after bulk culture stimulation with PMA and ionomycin in all 3 murine models of LN (Figure 2, A-D, Supplemental Figure 2A). There was also a reduced frequency of IL- $10^{+}$producin $\mathrm{CD} 4^{+} \mathrm{KITs}$ compared with that in splenic $\mathrm{T}$ cells. In addition to a reduced percentage of cytokine-producing cells among KITs compared with splenic T cells, there was also a substantial reduction in mean fluorescence intensity (MFI) of cytokine expression (Figure 2, C and D). KITs from B6 mice displayed a similar low frequency of cytokine-producing cells (Supplemental Figure 3A). There was no detectable IL-4 or IL-17 staining in KITs or splenic-derived T cells in the MRL/lpr model (not shown).

Both $\mathrm{CD} 4^{+}$and $\mathrm{CD} 8^{+} \mathrm{MRL} / \mathrm{lpr}$-derived KITs exhibited reduced proliferation in response to combined anti-CD3/anti-CD28 stimu- 
A

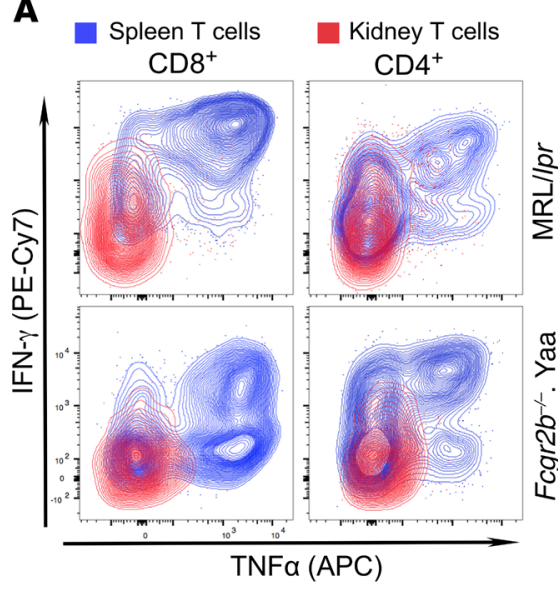

B

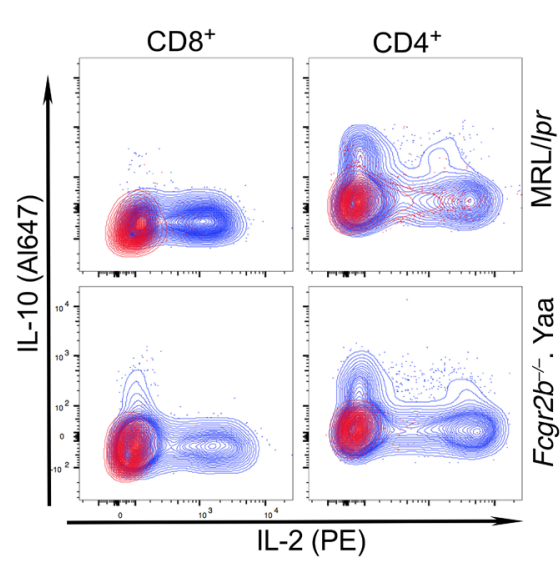

E
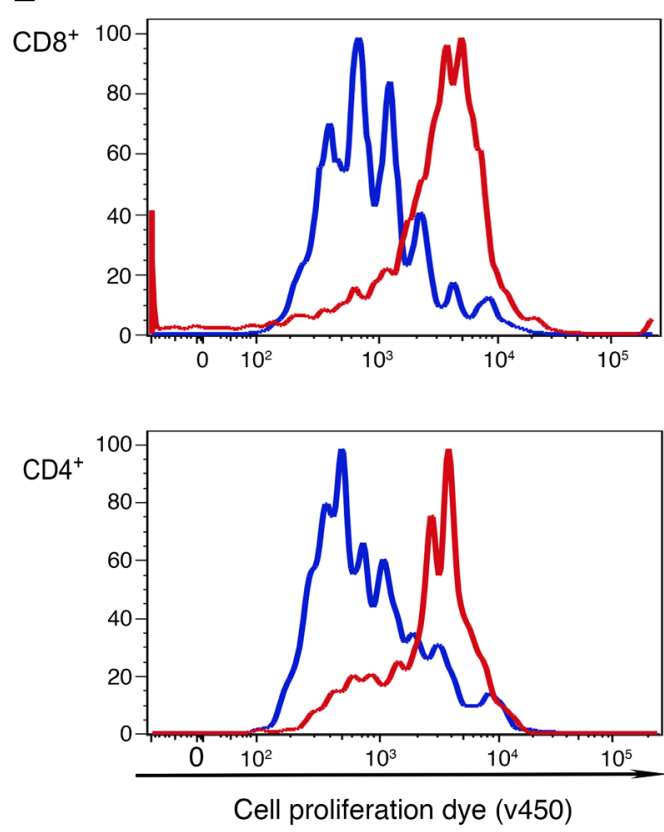

C

D
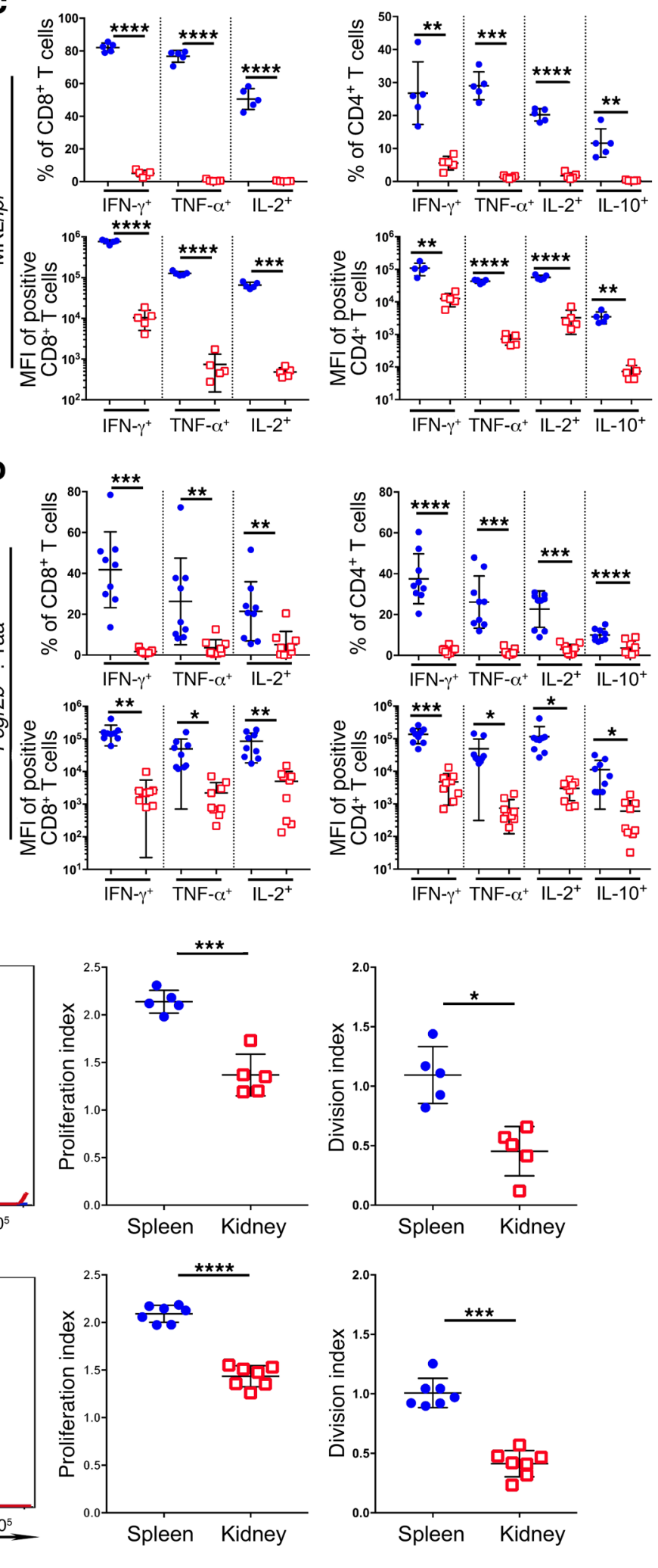
Figure 2. KITs have suppressed functional capacity. T cells were isolated from the kidney (red) and spleen (blue) of nephritic MRL/Ipr and Fcgr2 $\mathrm{b}^{-1-}$ Yaa mice. (A-D) Cells were stimulated in bulk culture with PMA and ionomycin in the presence of brefeldin $A$ for 4 hours, and cytokine expression was assessed by flow cytometry. (A and B) Representative contour plots showing cytokine production by $\mathrm{CD} 4^{+}$and $\mathrm{CD8}{ }^{+} \mathrm{T}$ cells from MRL/Ipr (upper panels)

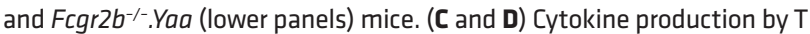
cells of MRL/Ipr (C) and Fcgr2b $b^{-1-}$.Yaa mice (D) represented as the percentage of positive cells (upper panels) and MFI of producers (lower panels). (E) Proliferation of $C D 8^{+} T$ cells in bulk culture after 3 days of anti-CD3/anti-CD28 stimulation (upper left panel) and 5 days for $\mathrm{CD} 4^{+} \mathrm{T}$ cells (lower left panel) from indicated organs. Cells were labeled with Cell Proliferation Dye prior to culture, and its staining is shown on the $x$ axis. Proliferation index and division index were calculated for $\mathrm{CD}^{+}(n=5)$ and $\mathrm{CD}^{+}(n=7)$ T cells. For tabulated data (C-E), each dot denotes an individual mouse and horizontal lines represent the mean, with error bars representing 1 SD. Paired Student's $t$ test was used to determine statistical significance between spleen and kidney samples. ${ }^{*} P<0.05$; ${ }^{* *} P<0.01 ;{ }^{* *} P<0.001$; ${ }^{* * *} P<0.0001$.

lation compared with splenic T cells, with only a few KITs proliferating past the first division, while splenic $\mathrm{T}$ cells underwent up to 6 divisions during the same time frame (Figure 2E). A significant reduction in both proliferative index and division index was observed (Figure 2E). Thus, counter to conventional theory, KITs are functionally suppressed, compared with activated effector splenic $\mathrm{T}$ cells.

KITs exhibit elevated levels of IRs. As functionally impaired KITs may be chronically exposed to self-antigen $(15,16)$, we hypothesized that they would exhibit an exhausted phenotype, as described for tumor-infiltrating $\mathrm{T}$ cells (TILs) and $\mathrm{T}$ cells in chronic infection $(35,36)$. To address this hypothesis, we examined expression of IRs known to be upregulated on exhausted $\mathrm{CD}^{+} \mathrm{T}$ cells, including PD-1, Tim3, Lag3, and 2B4 (35-37). Overall, CD8 ${ }^{+}$KITs from $\mathrm{MRL} / \mathrm{lpr}$ and $\mathrm{Fcgr} 2 \mathrm{~b}^{-/ .}$.Yaa LN mouse models exhibited increased IR expression compared with B6 splenocytes (Figure 3). Additionally, IR expression was generally more elevated in KITs than matched splenic comparators. This was most notable in the CD8 $8^{+}$ compartment in MRL/lpr and MRL.Tlr9-/- mice, with all IRs being expressed on a significantly higher proportion of KITs compared with their splenic counterparts (Figure 3A and Supplemental Figure 2B). A similar pattern of extensive expression of IRs was noted in the $\mathrm{Fcgr}_{2} \mathrm{~b}^{-/ . Y a a}$ KITs when compared with nonlupus control B6 $\mathrm{T}$ cells (Figure $3 \mathrm{~A}$ ). However, splenic $\mathrm{CD} 8^{+} \mathrm{T}$ cells in this model expressed IRs to a degree similar to that of KITs, possibly reflecting a high activation status of the $\mathrm{Fcgr}_{2} \mathrm{~b}^{-1}$.Yaa splenic T cells, as activation per se can also cause increased expression of IRs $(36,37)$.

Analysis of IR expression on CD4 ${ }^{+} \mathrm{T}$ cells is more complex, in part because the association between IR expression and exhaustion versus activation is less clear in $\mathrm{CD} 4^{+} \mathrm{T}$ cells (38). Nonetheless, we observed a statistically significant increase in the majority of IRs in KITs compared with nonautoimmune comparator T cells (Figure 3B). Notably, there were concomitant increases in the Lag3 and Tim3 expression in the splenic-derived MRL/lpr CD4 ${ }^{+}$ $\mathrm{T}$ cells, which is likely due to the activated nature of these circulating T cells (38). Both $\mathrm{CD}^{+}$and $\mathrm{CD} 8^{+}$KITs isolated from $\mathrm{B} 6$ mice exhibited substantially increased expression of PD- 1 compared with matched splenic controls. However, these B6-derived KITs expressed significantly less PD-1, Lag3, and Tim3 than KITs derived from lupus-prone MRL/lpr mice (Supplemental Figure 4).
Nearly all KITs exhibited suppressed cytokine production (Supplemental Figure 5, A and B), independent of PD-1 expression levels, suggesting that there are likely additional mechanisms by which KIT function is suppressed. This hypothesis is further supported by the fact that $\mathrm{T}$ cells derived from nonautoimmune kidneys did not produce cytokines after stimulation (Supplemental Figure 3) despite the fact that they expressed a significantly lower frequency of IRs than did KITs from autoimmune mice (Supplemental Figure 4).

Autoimmune KITs are metabolically quiescent compared with splenic-derived $T$ cells. Exhausted $\mathrm{T}$ cells are metabolically dysfunctional, as shown by reduced mitochondrial capacity, mitochondrial function, and glucose uptake (39). In fact, it has been suggested that metabolic dysfunction may be a mechanistic contributor to the exhaustion phenotype $(39,40)$. Thus, we subsequently assessed the mitochondrial reserve and metabolic output of KITs using the Seahorse Extracellular Flux Analyzer. Metabolic flux analysis of both $\mathrm{CD}^{+}$and $\mathrm{CD} 8^{+} \mathrm{KITs}$ from MRL/lpr mice revealed a substantial loss of spare respiratory capacity (SRC), defined as the difference between basal and uncoupled maximal oxygen consumption (Figure 4A). However, basal oxygen consumption rates were unchanged between cell types.

A reduction in SRC can be explained by loss of mitochondrial membrane potential $(\Delta \psi \mathrm{m})$ and/or loss of mitochondrial mass. Because $\Delta \psi \mathrm{m}$ is required for production of ATP, it has been hypothesized that suppressed $\Delta \psi \mathrm{m}$ may alter cellular function (40). Indeed, using MitoStatus, a membrane potentialdependent dye, we observed that KITs from MRL/lpr, $\mathrm{Fcgr}_{2} \mathrm{~b}^{-/}$. $Y a a$, and MRL.Tlr $9^{-/-}$mice exhibited reduced $\Delta \psi \mathrm{m}$ compared with splenic counterpart cells (Figure 4B and Supplemental Figure 2C). Similarly, both $\mathrm{CD}^{+}$and $\mathrm{CD} 8^{+}$KIT populations had a significant $(P<0.01$ for all comparisons) reduction in MitoTracker Deep Red FM (MitoTracker) staining, which measures mitochondrial mass (39). However, CD8 $8^{+}$KITs had a bimodal distribution of MitoTracker staining (Figure $4 \mathrm{C}$ and Supplemental Figure 2D), with some cells retaining substantial staining. Thus, the loss of mitochondrial mass appears to contribute to, but alone is insufficient to account for, the almost complete abrogation of SRC.

The extent of glucose utilization depends on $\mathrm{T}$ cell function $(39,41)$, with exhausted $\mathrm{T}$ cells exhibiting reduced glucose uptake (39). Indeed, KITs from MRL/lpr mice demonstrated suppressed glucose uptake in vitro, as measured by reduced fluorescent 2-NBD-glucose (2NBDG) dye uptake. In MRL/lpr and MRL.Tlr9--- mice, $25.7 \%$ and $30.0 \%$ of $\mathrm{CD} 8^{+}$KITs took up glucose, respectively, compared with $86.2 \%$ and $67.8 \%$ of splenic comparators (Figure 4D and Supplemental Figure 2C). Furthermore, in costaining studies, very few KITs in either the $\mathrm{CD} 4^{+}$or $\mathrm{CD}^{+}$compartment exhibited intact $\Delta \psi \mathrm{m}$ and glucose uptake (Figure 4D), suggesting that these cells carry out little if any oxidative phosphorylation (Figure 4D). MRL.Tlr9-/- KITs (Supplemental Figure 2, C and D) and T cells derived from B6 kidneys (Supplemental Figure 3B) exhibited a similar pattern of mitochondrial probe and 2NBDG staining. One notable difference was that $\mathrm{CD} 4^{+}$cells isolated from kidneys of $\mathrm{B} 6$ mice did contain a population of metabolically intact cells (MitoStatus and 2NBDG double positive). Among both KITs and splenic T cells, only those cells that had not upregulated PD-1 demonstrated 
A
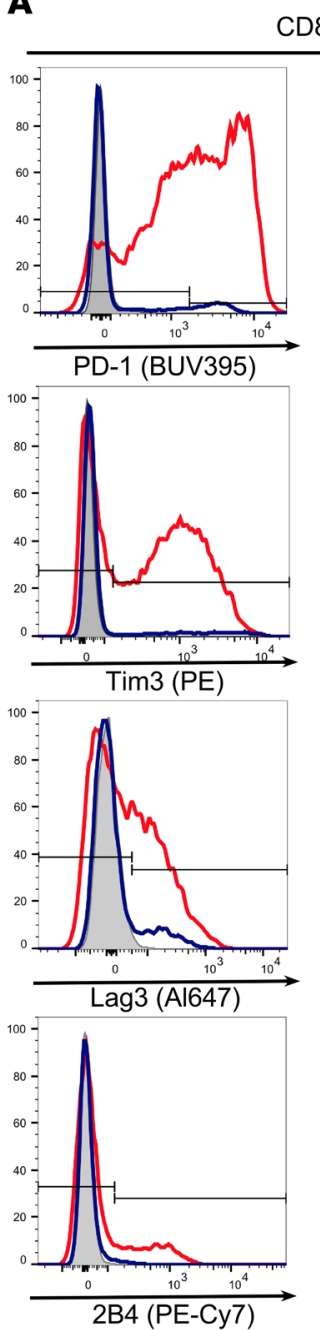

$\mathrm{CD}^{+}$
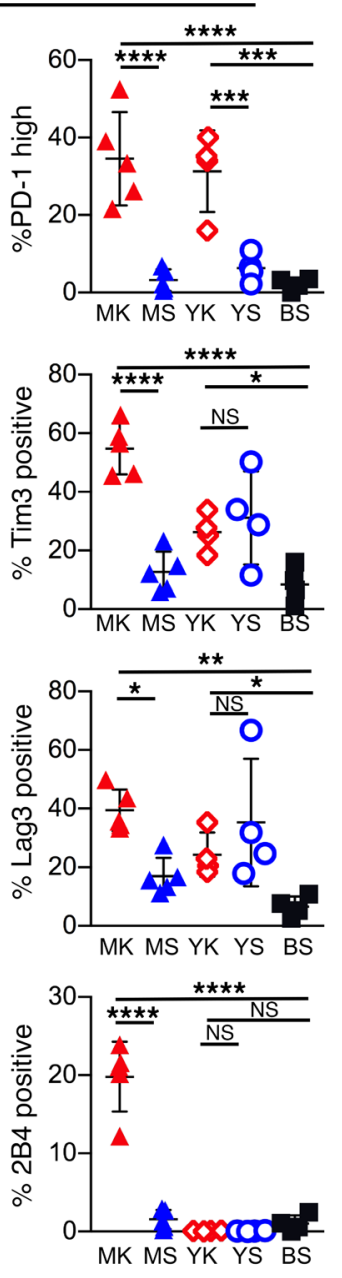

B
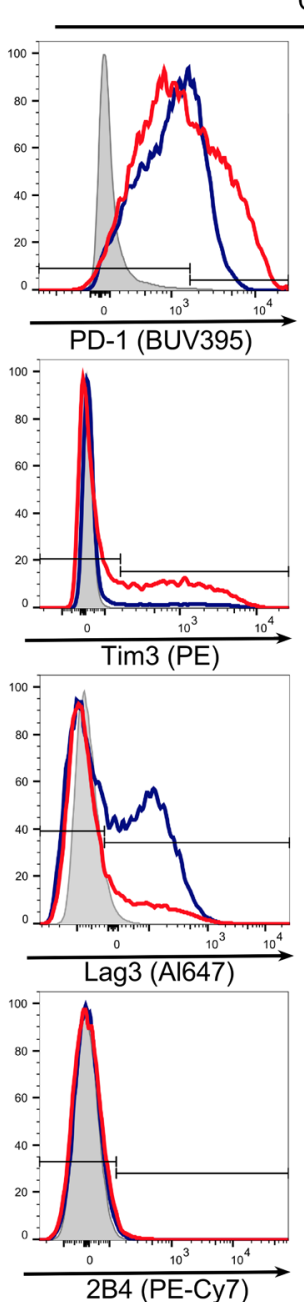

$\mathrm{CD}^{+}$
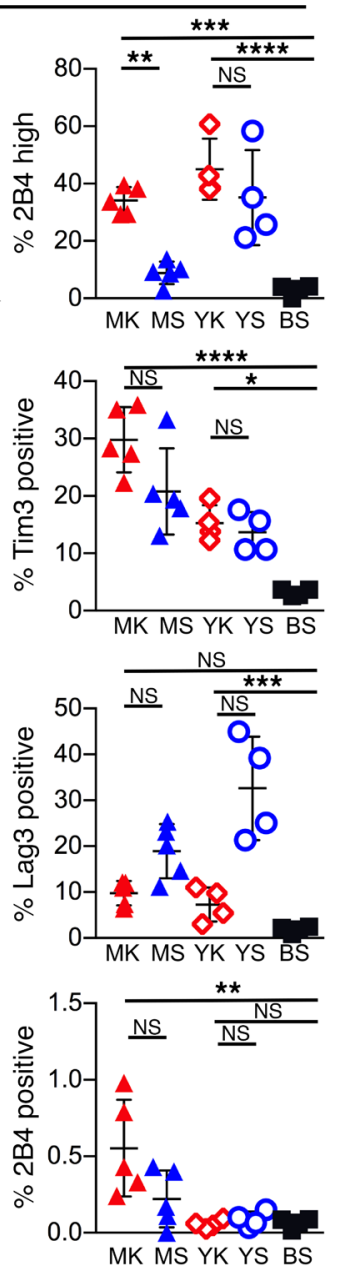

Figure 3. KITs increase expression of IRs. Representative histograms of IR expression on CD8+ (A) and CD4+ (B) T cells from kidney (red) and spleen (blue) of MRL/Ipr and B6 (gray) mice, with gating parameters (left column). Right columns show summary data from MRL/lpr (solid symbols, $n=5 / \mathrm{group}$ ), Fcgr2b-1.Yaa (open symbols, $n=4$ /group), and B6, nonlupus controls (black, $n=4$ /group). For tabulated data, each dot denotes an individual mouse, and horizontal lines represent the mean, with error bars representing 1 SD. One-way ANOVA with Tukey's multiple comparison was performed. ${ }^{*} P<0.05$; ${ }^{* *} P<$ $0.01 ;{ }^{* *} P<0.001 ;{ }^{* * *} P<0.0001$. These findings are representative of 3 replicates of MRL//pr mice and 2 replicates of $F c g r 2 b^{-1-}$.Yaa mice.

glucose uptake (Supplemental Figure 5C), but neither mitochondrial parameter had a similar correlation with PD-1 expression (Supplemental Figure 5, D and E), again suggesting that PD-1 expression and function alone do not entirely control the metabolic exhaustion phenotype in these cells.

KITs from younger MRL/lpr mice have a phenotype similar to that of nephritic MRL/lpr mice. To begin to address when the T cell exhaustion phenotype observed in KITs of aged mice first develops, we examined a cohort of 11- to 12-week-old MRL/lpr mice, at a time when disease is at early stages and prior to clinical proteinuria. KITs derived from 11- to 12-week-old MRL/lpr mice did not differ significantly from those of aged nephritic MRL/lpr mice with regard to activation status, expression of exhaustion markers, cytokine production, and metabolic profile (Supplemental Figures 1,3 , and 4). This finding suggests that alteration in $\mathrm{T}$ cell profile after kidney infiltration occurs fairly early in the disease process and may occur relatively rapidly after tissue entry.
KITs exhibit a transcriptional phenotype that resembles "exhausted" $T$ cells from other models of disease. To better understand how KITs and splenic T cells from the same animals differed in function and origin, we performed transcriptional profiling of $\mathrm{CD} 4^{+}$ and $\mathrm{CD}^{+}$KITs and matched splenic T cells. After determining genes that were differentially expressed between splenic $\mathrm{T}$ cells and KITs (42), we performed gene set enrichment analysis (GSEA) (43). Differentially expressed genes were compared with several previously defined gene signatures that are characteristic of $\mathrm{CD} 8^{+}$ and $\mathrm{CD} 4^{+} \mathrm{T}$ cell exhaustion in the chronic lymphocytic choriomeningitis virus (LCMV) infection model $(37,44)$. Among genes that were differentially expressed in $\mathrm{CD} 8^{+} \mathrm{KITs}$ versus $\mathrm{CD} 8^{+}$splenocytes, genes from the $\mathrm{CD} 8^{+}$exhaustion cluster were significantly enriched (Figure 5A and Supplemental Figure 6). Similarly, among genes differentially expressed in $\mathrm{CD} 4^{+} \mathrm{KITs}$ versus $\mathrm{CD} 4^{+}$splenocytes, genes contained in the $\mathrm{CD} 8^{+} \mathrm{LCMV}$ exhaustion-associated gene set were significantly enriched (Supplemental Figure 6), and 
A

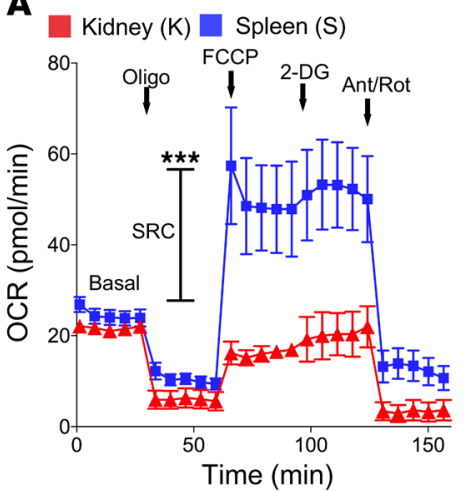

CD4+

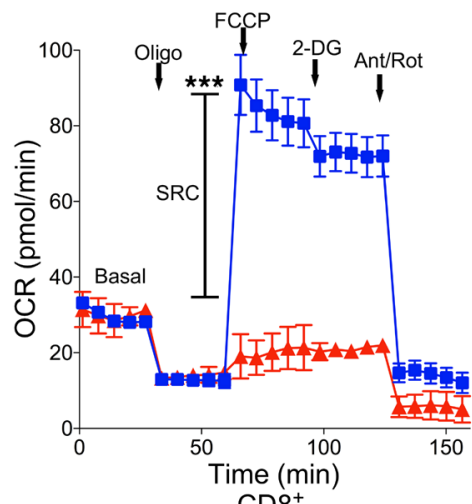

$\mathrm{CD} 8^{+}$

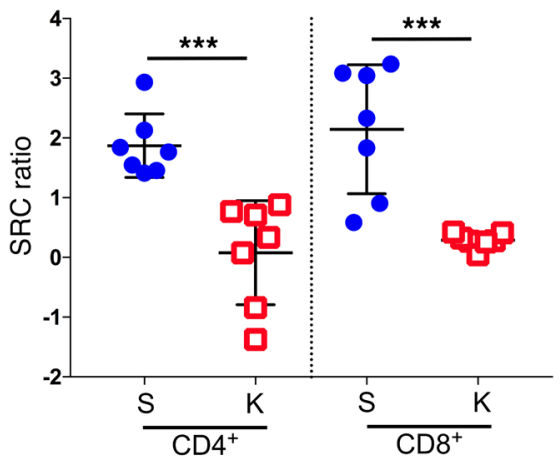

C

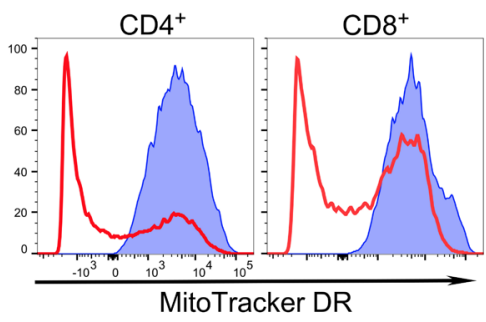

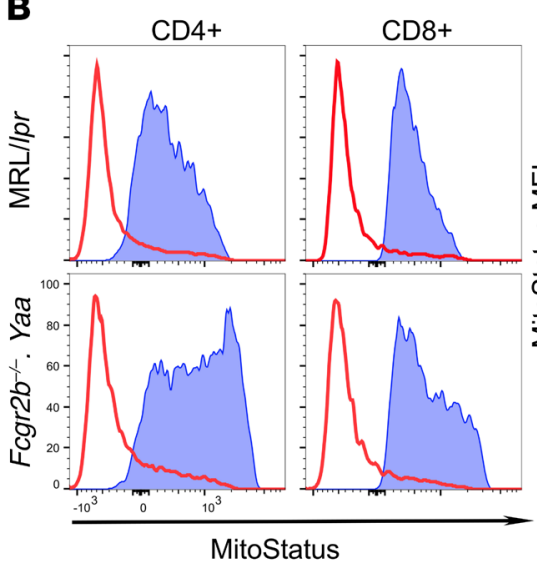
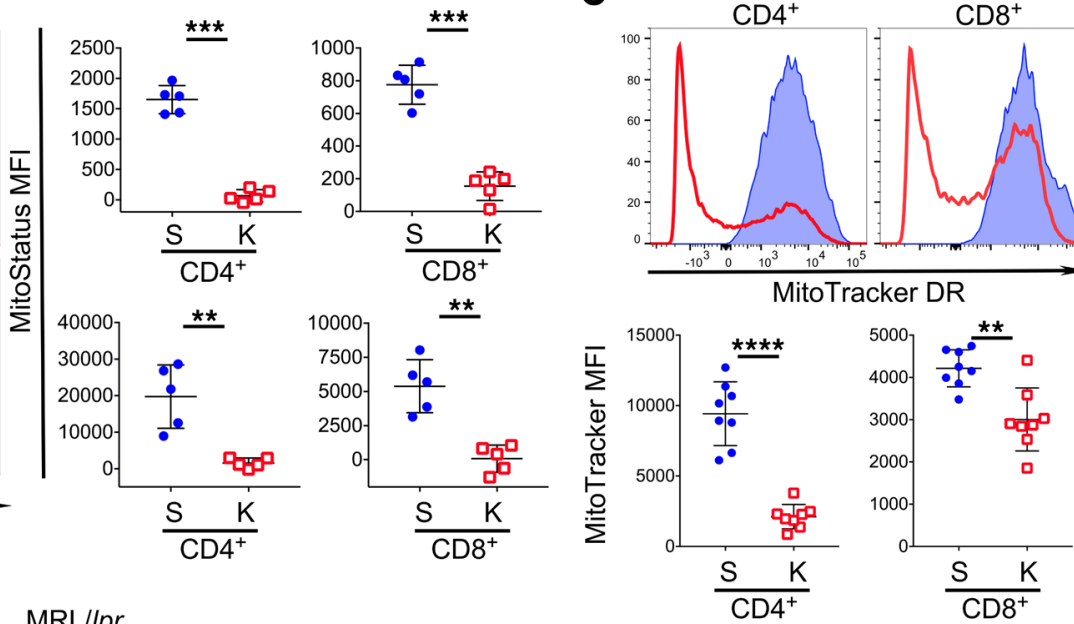

D
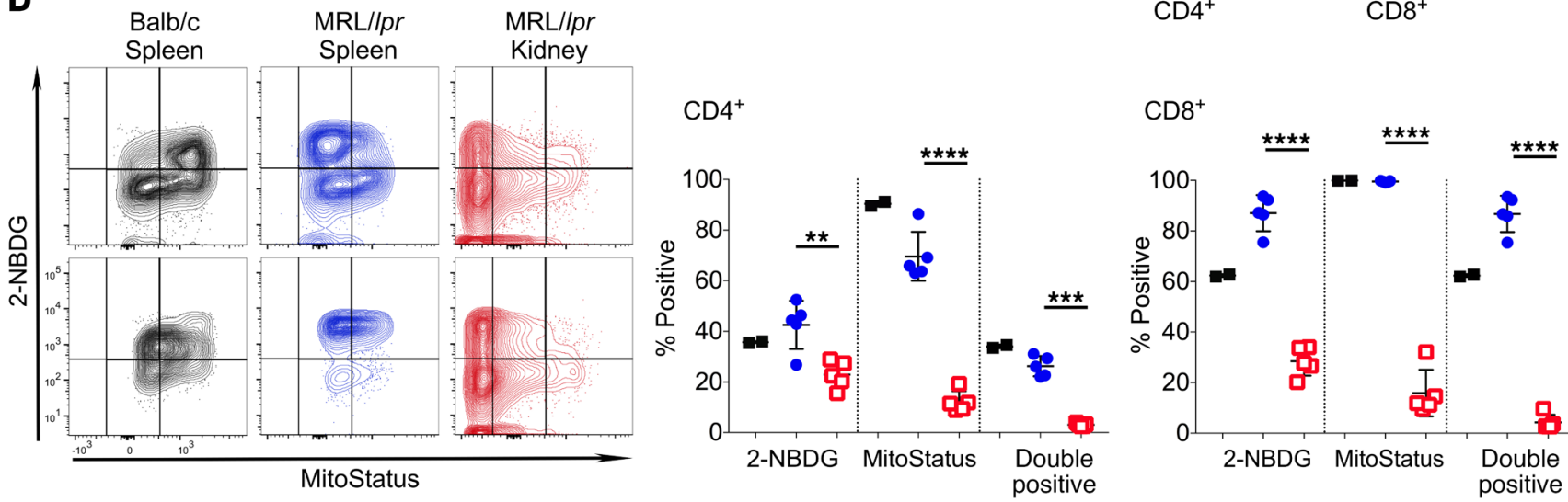

Figure 4. KITs are metabolically suppressed. (A) Representative oxygen consumption rate (OCR) trace (left) from sorted CD4+ and CD8 $8^{+} \mathrm{T}$ cells isolated from kidney (red) and spleen (blue) of MRL//pr mice. A metabolic stress test was performed by injection of oligomycin (Oligo), mitochondrial decoupler (FCCP), glucose uptake inhibitor (2-DG), and antimycin A/rotenone (Ant/Rot). SRC was calculated as the difference between basal OCR values and maximal OCR values achieved after FCCP uncoupling. Summary data of SRC ratio, defined as SRC divided by basal OCR, is shown on the right ( $n=7$ per group). Error bars at each time point in the trace represent mean \pm SEM of triplicate wells, with tabulated data in dot plots (right panels). (B) The $\Delta \psi m$ was assessed by flow cytometry using MitoStatus dye. Representative histograms (red, kidney; blue, spleen) of MitoStatus from T cell lineages as indicated from both MRL/Ipr (upper left panel) and Fcgr2b-/-Yaa mice (lower left panel), with tabulated data in dot plots (right panels) ( $n=5$ per group), are shown (C) Mitochondrial mass was assessed by flow cytometry using MitoTracker DR. Representative histograms (red, kidney; blue, spleen) of MitoTracker DR from T cell lineages as indicated from MRL/Ipr mice with summary data in dot plots (lower panels) ( $n=5$ per group). (D) Representative contour plots of BALB/c splenic or MRL/Ipr splenic- or kidney-derived CD4+ (top) and CD8+ (bottom) T cells showing 2NBDG (glucose uptake) and MitoStatus staining, with summary data in dot plots (BALB/c, $n=2 ; M R L / I p r ~ n=5$ mice). For tabulated data (B-D), each dot denotes an individual mouse, and horizontal lines represent the mean, with error bars representing 1 SD. Paired Student's $t$ test was used to determine statistical significance between spleen and kidney samples. ${ }^{* *} P<0.01 ;{ }^{* *} P<0.001 ;{ }^{* * *} P<0.0001$. 
A

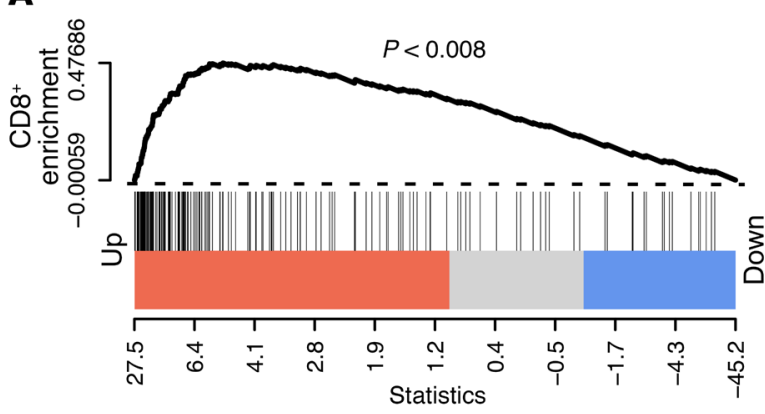

B

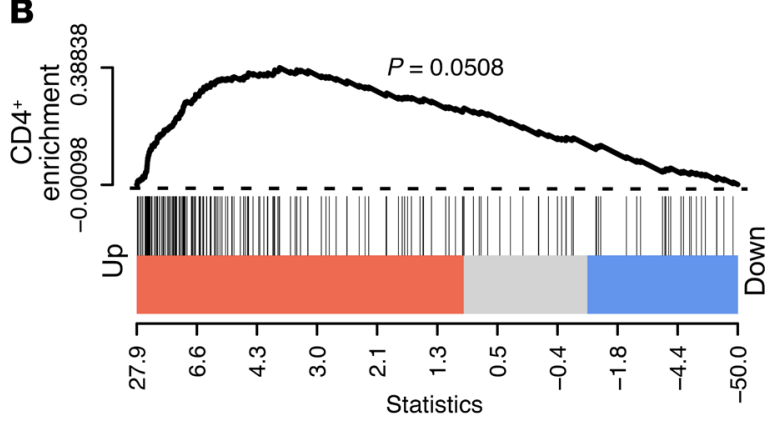

C Row annotation $\square$ TIL

$\square$ Naive $\square$ Mixed
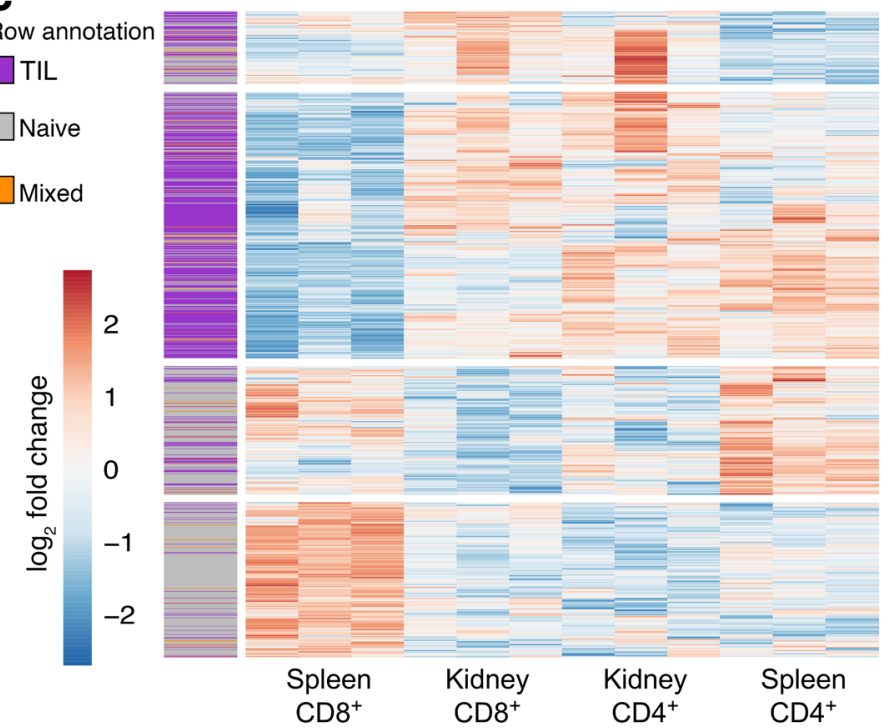

D

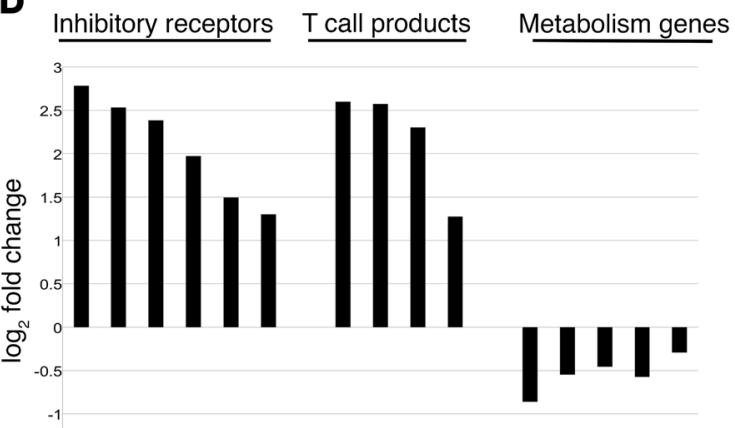

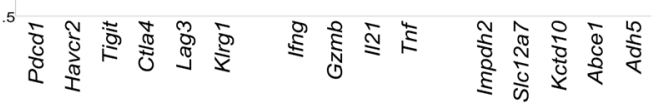

E

Control T cells

$\square$ Exhausted T cells

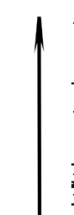

:

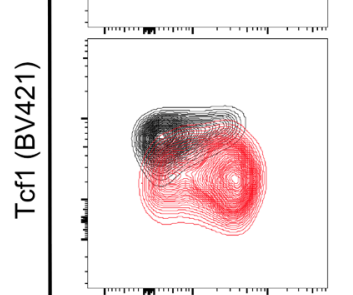

Kidney $T$ cells

Splenic T cells
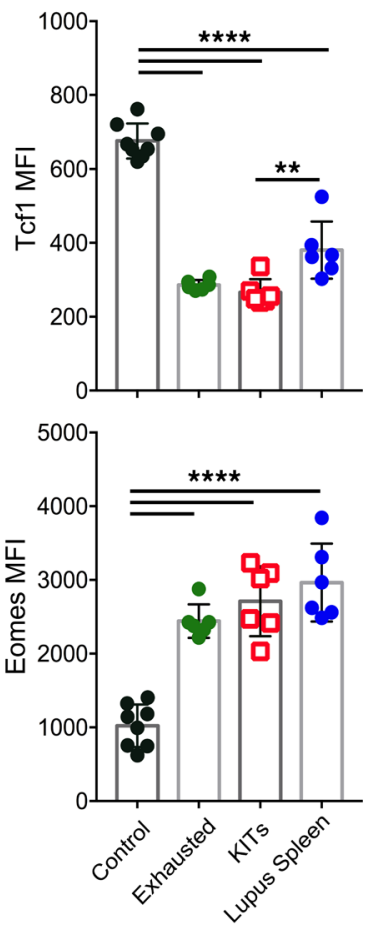

Figure 5. The transcriptional profile of KITs is consistent with T cell exhaustion. (A and B) RNA-seq data were used to construct gene set enrichment plots illustrating genes differentially regulated in kidney- compared with splenic-derived T cells ( $n=3$ per group) with respect to a known set of 194 CD4 expressed and $200 \mathrm{CD} 8^{+}$expressed genes specific for LCMV-induced T cell exhaustion (44) in both the CD8 ${ }^{+}$(A) and CD4 $4^{+}$(B) compartments. MitoStatus values calculated using the conservative rankSumTestWithCorrelation function in the limma package. (C) Unbiased hierarchical clustering was performed using 1,426 genes previously identified from 10 clusters comparing TILs to activated and naive T cell populations (36) and represented as a heatmap. Row annotation on the heatmap shows association of clustered genes with PD-1+Tim3 $3^{+}$TILs (purple), naive, effector, and PD- $1^{-}$Tim3 $3^{-}$TILs (gray) or those with enhanced gene expression in both subgroups (orange) (36). GSEA was also performed on individual clusters (Supplemental Figure 6). (D) Differential expression between kidney and splenic CD8 ${ }^{+} \mathrm{T}$ cells for selected IRs, T cell products, and metabolic genes as determined by RNA-seq. (E) Representative contour plots for Tcf1 and Eomes expression in nonexhausted (black), exhausted (PD-1+ Tim3 ${ }^{+}$T cells from LCMV-infected mice) (green), or MRL/lpr kidney(red) or splenic-derived (blue) $\mathrm{CD}^{+} \mathrm{T}$ cells. For tabulated data, each dot denotes an individual mouse, and bars represent the mean, with error bars indicating SD. One-way ANOVA was used to determine statistical significance using Tukey's test for multiple comparisons. ${ }^{* *} P<0.01 ;{ }^{* * * *} P<0.0001$.

among CD $4^{+}$KIT differentially expressed genes, genes characterizing exhausted $\mathrm{CD} 4^{+} \mathrm{T}$ cells in LCMV were nearly significantly enriched $(P=0.0508$, Figure $5 \mathrm{~B})$. These data provide direct and unbiased evidence that $\mathrm{CD} 8^{+} \mathrm{KITs}$ are more exhausted than their lymphoid-resident counterparts. The less robust enrichment of exhaustion-related genes among those differentially expressed by $\mathrm{CD}^{+}{ }^{+} \mathrm{KITs}$ could have several explanations: there may be a component of peripheral lymphoid exhaustion in the $\mathrm{CD} 4^{+}$compartment of older diseased mice we studied. In this case, the differential expression analysis between the kidney and spleen would 

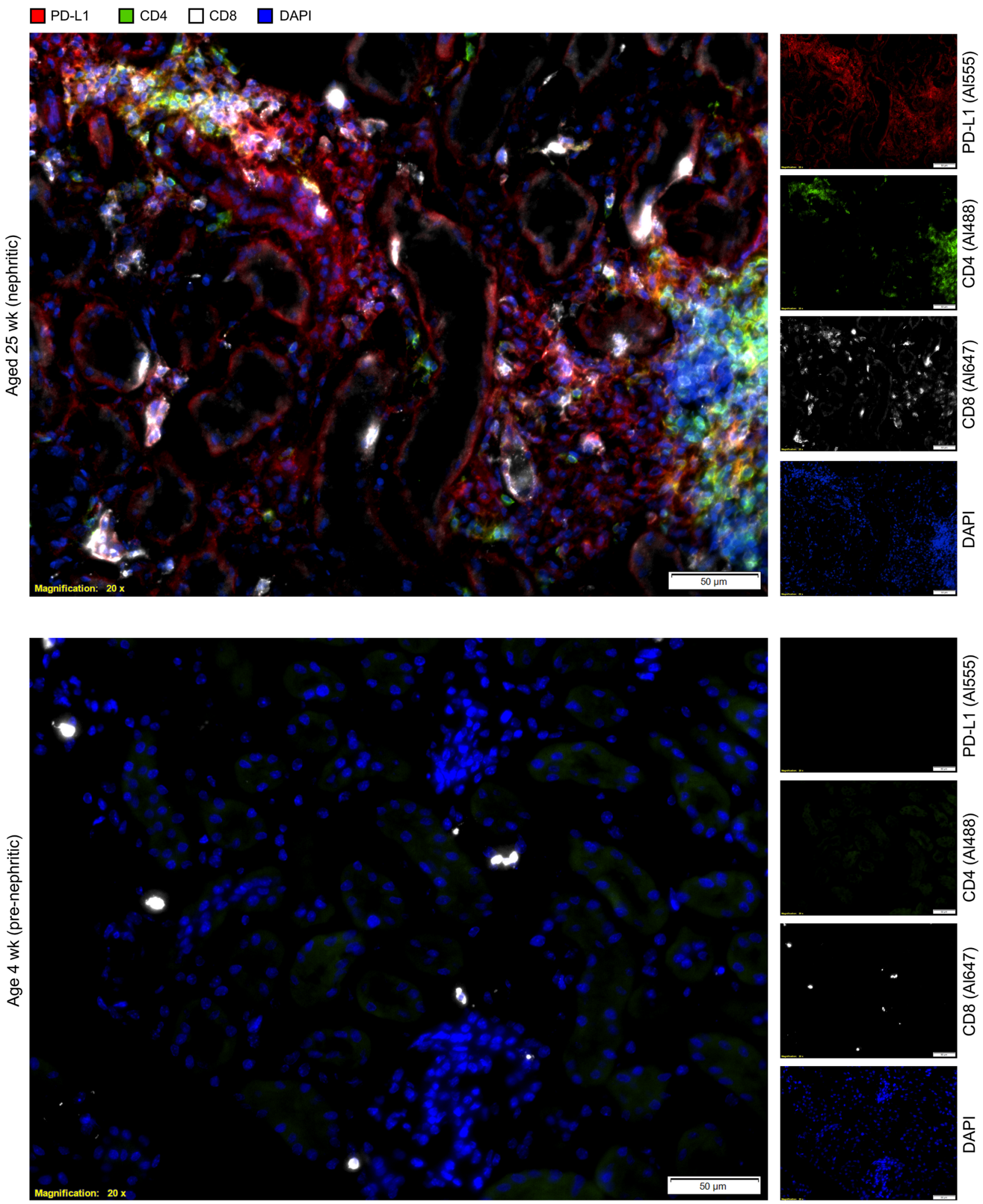

Figure 6. Nephritic kidneys from MRL/Ipr mice locally express PD-L1 near sites of T cell infiltration. PD-L1 expression on kidneys of nephritic (>20 weeks) and prenephritic (4 weeks) MRL/lpr mice was assessed by immunofluorescence microscopy: CD4+ (green), CD8 (white), and PD-L1 (red) expression. Merged and single-color images are shown for each stain. Scale bars: $50 \mu \mathrm{m}$ (insets). 
filter out some exhaustion-related genes. Related to this, highly activated cells are documented to turn on genes associated with exhaustion (36), and our splenic CD4 ${ }^{+}$population expressed multiple exhaustion-associated IRs and is $\mathrm{CD}_{4} 4^{+}$and $\mathrm{CD} 69^{+}$(Figures 1 and 3). Additionally, $\mathrm{CD} 4^{+}$exhaustion is less well defined than that of $\mathrm{CD}^{+}$cells (35).

We also compared our data sets to the exhaustion profile from a murine model of melanoma (36). Ten gene clusters were identified in this prior study by comparing naive, effector, and IRexpressing tumor-infiltrating $\mathrm{CD}^{+}$cells. Four of these clusters (most predominantly $\mathrm{C} 1$ and $\mathrm{C} 2$ and, to a lesser extent, C7 and C10) were associated with PD- $1^{+}$Tim $3^{+}$TILs (commonly linked with exhaustion), while 4 clusters (C3, C4, C5, and C6) were associated with phenotypically naive or effector populations, and 2 clusters showed a mixed pattern (C8 and C9). A total of 1,426 differentially expressed genes between $\mathrm{CD} 4^{+}$and $\mathrm{CD} 8^{+} \mathrm{KITs}$ and splenic $\mathrm{T}$ cells from our MRL/lpr mice were delineated from a total of 3,031 possible genes present in any the 10 clusters. Notably, unbiased hierarchical clustering of these 1,426 genes resulted in correlation of expression between the TIL-related genes and both $\mathrm{CD}^{+}$and $\mathrm{CD}^{+} \mathrm{KIT}$ differentially expressed genes (Figure 5C). GSEA further supported the finding that the CD8 ${ }^{+}$KITs are similar to $\mathrm{PD}-1^{+} \mathrm{Tim}^{+}$TILs, with significant positive correlation for gene clusters $\mathrm{C} 1$ and $\mathrm{C} 2$ and negative correlation for the naive cell clusters C4 and C5 (Supplemental Figure 7).

$\mathrm{CD}^{+}$KITs exhibited increased transcription of IRs, in agreement with the flow cytometry data (Figure 3 and Figure 5D), and decreased transcription of metabolism-related genes defined in LCMV exhaustion (37). Interestingly, $\mathrm{CD}^{+}$exhausted cells expressed increased levels of inflammatory cytokine transcripts, while expression of these proteins was actually reduced (Figure 2 and Figure 5D). The finding of increased transcripts by RNA sequencing (RNA-seq) is in line with microdissection studies of KITs in LN that revealed increased cytokine transcripts $(14,18)$. The phenomenon of discordant mRNA and protein levels is well documented (45), particularly for cytokines that undergo significant posttranscriptional regulation $(46,47)$. Importantly, this regulation may play an integral role in T cell exhaustion in KITs, as a similar discordant protein/transcript phenomenon was previously observed for LCMV-specific CD8 ${ }^{+} \mathrm{T}$ cells (37).

Exhausted CD8 ${ }^{+} \mathrm{T}$ cells express increased Eomes (48) and decreased Tcf1 (49). Thus, we next examined whether the expression pattern of the transcription factors Eomes and Tcf1 in KITs mirrored that of $\mathrm{T}$ cell exhaustion in the LCMV model of chronic viral infection. KITs were compared with PD- ${ }^{\text {hi }}$ Tim $3^{\text {hi }}$ (exhausted) $\mathrm{T}$ cells from LCMV-infected mice and control nonexhausted cells. Notably, KITs expressed a Tcf1 and Eomes profile similar to that of exhausted T cells after LCMV infection (Figure 5E), with significantly decreased Tcfl expression compared with control (nonexhausted T cells) and splenic-derived T cells from matched $\mathrm{MRL} / \mathrm{lpr}$ mice and a concomitant increase in Eomes compared with that in nonexhausted $\mathrm{T}$ cells (Figure 5E). This suggests that the transcriptional phenotype observed in KITs may be mediated by mechanisms similar to those regulating $\mathrm{T}$ cell exhaustion in chronic viral infections.

$P D-L 1$ expression is increased in LN kidney compared with noninflamed kidney. Several studies suggest that the PD-L1/PD-1 sig- naling axis is protective in murine lupus (50-52). These studies relied on global gene deletion, which would have affected activation of cells in secondary lymphoid tissues as well as target organs. Thus, to begin to determine how $\mathrm{T}$ cell exhaustion is mediated in nephritic kidneys, we investigated in situ PD-L1 expression in kidneys of diseased MRL/lpr mice. T cell infiltrates were observed both in the periglomerular space and the interstitium of nephritic mice (Figure 6 and Supplemental Figure 8), but not in younger, prediseased mice (Figure 6). PD-L1 was highly expressed in 4 of 6 nephritic mice on both parenchymal and infiltrating cells and in 2 of 6 mice on predominantly infiltrating cells, while none of the control, prediseased MRL/lpr mice had any PD-L1 staining (Figure 6 and Supplemental Figure 8). Flow cytometric analysis showed that the majority of infiltrating cells expressing elevated PD-L1 levels were $\mathrm{CD}_{11 \mathrm{~b}^{+}}$(Supplemental Figure 9). The finding of PD-L1 expression in murine nephritic kidneys parallels two studies of human LN kidneys, which showed increased PD-L1 in biopsy samples $(53,54)$. Hence, in the context of autoimmune nephritis, PD-L1 is induced in both mice, based on our data, and SLE patients, based on published studies, providing a potential mechanism for induction of an exhausted-like T cell phenotype.

\section{Discussion}

A paradigm in the field of autoimmunity is that aggressive, activated self-reactive $\mathrm{T}$ cells infiltrate tissue and destroy target organs. Nonetheless, there has been little direct characterization of tissueinfiltrating cells in disease, while cells found in blood and lymphoid organs have been extensively studied. Herein, we present an in-depth characterization of tissue-infiltrating $\mathrm{T}$ cells in the kidneys of mice with lupus-like nephritis. Our central and most unexpected finding is that the great majority of KITs are not activated effector cells, as had been thought; rather, they demonstrate suppressed cytokine production and proliferative capacity, with enhanced expression of IRs. In a potential mechanistic link, these cells have reduced metabolic flux, reduced SRC, and reductions in mitochondrial numbers and function. Perhaps most tellingly, we observed that the transcriptional profile of KITs mimicked the T cell exhaustion profile observed in other contexts $(36,37)$. Taken together, the data indicate that KITS closely resemble $\mathrm{T}$ cells found in chronic infection and infiltrating tumors $(35-37,44)$.

A central concept in tumor immunology is that the tumor microenvironment actively suppresses the function of invading $\mathrm{T}$ cells through specific adaptive mutations and increased expression of ligands for IRs (55). It may have been assumed, therefore, that nonmalignant parenchymal tissues generally lacked the capacity to suppress infiltrating cells or, at the least, that such capacity was compromised to permit autoimmunity. The conceptual advance driven by our current study is that normal parenchymal tissue has the ability to suppress $T$ cells in a fashion similar to that of tumors, even in the context of genetic predisposition to and clinical existence of autoimmunity. We suggest that target tissues may not be as susceptible to autoimmune infiltrates as previously thought and may be endowed with multiple mechanisms to naturally suppress locally destructive adaptive immunity, with these mechanisms still operating or even enhanced in the face of ongoing autoimmune attack. Given that the KIT exhausted phenotype, as we described herein, correlates well with that of tumor-infiltrating lymphocytes, 
we hypothesize that it may reflect the existence of a general phenotype of $\mathrm{T}$ cells infiltrating parenchymal tissues during inflammation. In this context, it is interesting to consider autoimmunity, which is observed in numerous organ systems, that emerges upon therapeutic inhibition of PD-1/PD-L1 in cancer immunotherapy (56). This complication may result from increased activation of autoreactive $\mathrm{T}$ cells in secondary lymphoid tissue; however, our data highlight another potential mechanism - that checkpoint inhibition could uncover latent autoimmunity by derepression of previously exhausted infiltrating cells that had been causing no clinically significant harm. This mechanism would also be consistent with the relatively rapid onset and aggressive nature of checkpoint blockade-induced autoimmunity (57).

Interestingly, transcriptional profiling revealed that TNF- $\alpha$ and IFN- $\gamma$ mRNAs were more highly expressed in the $\mathrm{CD}^{+}$KITs compared with the effector splenic $\mathrm{CD} 8^{+} \mathrm{T}$ cell population, whereas protein expression was just the opposite, with KITs expressing much less than peripheral effectors. Though seemingly paradoxical, it is actually not surprising, as a similar phenomenon was described for LCMV-driven T cell exhaustion (37). Our data and these prior data on LCMV-driven exhausted CD8 ${ }^{+} \mathrm{T}$ cells together imply that a major mechanistic checkpoint for enforcement of reduced effector function by exhausted cells is via posttranscriptional regulation of cytokine, and perhaps other, genes. How this takes place has yet to be determined. In any case, these data suggest that $\mathrm{T}$ cell exhaustion and suppression of cytokine signaling is an active process and not just another form of anergy.

How parenchymal tissues under autoimmune attack respond to inhibit the functional status of infiltrating $\mathrm{T}$ cells is not yet clear and is likely to be at least as complex as understanding mechanisms at play in tumors, an area of long-term and extensive research that still is not fully resolved. Nonetheless, from the tumor and chronic viral infection fields, signaling via PD-1 has emerged as a major, but nonexclusive, axis that enhances the exhausted phenotype. Based on this, we looked for, and found, local expression of PD-L1 on infiltrated but not noninfiltrated kidneys in MRL/lpr mice, in agreement with published studies of LN in humans $(53,54)$. Interestingly, PD-L1 expression occurs in close proximity to infiltrating $\mathrm{T}$ cells, which suggests that parenchymal tissues sense inflammatory signals from infiltrating $\mathrm{T}$ cells and respond by upregulating PD-L1 (and potentially other inhibitory ligands). The importance of PD-L1 upregulation is further supported by prior studies that show that PD-L1 expression on kidney-derived tubular epithelial cells lines can suppress $\mathrm{T}$ cell function $(58,59)$. A similar relationship between infiltrating cells and PD-L1 expression has been described in tumors (60). If it is true that PD-L1 in parenchymal tissue acts to surpress infiltrating cells, this would represent a tissue adaptation to inflammation manifesting even in an autoimmune-prone mouse.

Given the profound phenotype of KITs observed in mice with active lupus-like disease, we examined the potential of tissue parenchyma to induce a $\mathrm{T}$ cell exhaustion phenotype in the absence of overt autoimmunity. KITs from B6 mice indeed have suppressed cytokine production and an altered metabolic profile similar to that observed in lupus-prone mice. Additionally, like $\mathrm{MRL} /$ pr KITs, these "nonlupus-prone"-derived kidney T cells differed substantially from splenic comparators, with an increased frequency of "effector cells" (CD44+, CD62L-) as well as increased frequencies of cells expressing both CD69- and PD-1. Yet they did not fully resemble KITs from MRL/lpr mice in several respects. Compared with MRL/lpr KITs, B6 KITs had lower frequencies of IR expression and expressed fewer overall IRs, contained a larger naive cell population, and had a metabolically intact subset of CD4 KITs. Given the 100-fold lower number of KITs infiltrating the kidney in B6 compared with MRL/lpr mice, the impact of these cells on tissue function is unknown. One hypothesis to explain these KITs and their partially exhausted phenotype is that they represent an age-dependent naturally occurring autoreactive population. In normal mice, we propose that the parenchyma and other factors successfully suppress these cells, resulting in no clinically apparent disease phenotype. Since the exhausted KIT phenotype is observed in B6 mice, it remains possible that this phenotype is not entirely dependent on the autoimmune/inflammatory microenvironment. Studies in which particular antigen-specific T cells are observed in either inflammatory or quiescent contexts would be needed to distinguish these possibilities, which are not mutually exclusive. Either scenario is consistent with the notion that the kidney possesses suppressive qualities, which, if confirmed, would have implications for hypertension, ischemia reperfusion injury, and renal transplantation.

A major question raised by these results is, Since T cells become exhausted in the target tissue, then how is damage mediated? In this context, it is important to note that exhausted T cells, while dysfunctional, are not inert. Exhausted T cells in infection play a nonredundant role in viral control despite their apparent exhaustion, as demonstrated by severe exacerbation of disease and death when these exhausted cells are experimentally depleted $(61,62)$. Hence, we suggest that, though most infiltrating cells are exhausted, they nevertheless retain some effector function and, in fact, cause slow rather than the acute destruction and decline that characterizes chronic autoimmunity in these LN models. Further, in these models, there are still some cells that do not display IRs and thus could conceivably not yet be exhausted. Thus, an additional or alternative model to explain disease pathogenesis in the face of exhaustion is that these IR-negative T cells, which could be newly arrived, initially cause damage and subsequently undergo exhaustion.

Given our knowledge about $\mathrm{CD}^{+} \mathrm{T}$ cell exhaustion in other settings, it is likely that there are, in addition to PD-1, other T cellintrinsic IRs that play a role in regulating lupus pathogenesis. We identified increased expression on KITs of Lag3, Tim3, and 2B4; whether these (or even PD-1) are functionally important and where the relevant ligands are expressed will need to be addressed in future work that ideally would involve tissue-specific genetic manipulation. It is important to distinguish tissue-localized exhaustion and PD-L1 expression from the regulation of autoimmune responses in general, which could emanate from initial or chronic activation in lymphoid tissues. Indeed, several reports demonstrate roles for PD-L1 in regulating autoimmune disease (50-52). These studies, which depended on global inactivation, hypothesized a role for PD-L1/PD-1 in peripheral $\mathrm{T}$ cell activation. Critically, prior genetic or inhibitor studies neither targeted nor characterized T cells in tissues.

Recent work has suggested that altered metabolism is a driving factor in T cell exhaustion $(39,40)$. As with TILs, KITs exhibited a markedly altered metabolic profile. Mitochondrial mass, flux capac- 
ity, and membrane potential are all suppressed in KITs. Furthermore, KITs demonstrate reduced glucose uptake, which may in part be responsible for suppression of cytokine production, since it is reported that suppression of aerobic glycolysis regulates translation of inflammatory cytokines (41). The kidney itself has high oxygen and glucose demand (63), and this may play a role in altering the metabolic profile of infiltrating $\mathrm{T}$ cells. Such a mechanism would be in keeping with our observation of reduced cytokine protein synthesis despite similar or increased gene transcription in KITs.

Another potential factor that could mediate exhaustion and inactivation of KITs is coinfiltrating CD11b cells, which were the second most numerous cell type in lupus kidneys and the most numerous cell type in nonlupus kidneys. These myeloid cells are diverse, based on initial flow cytometric analysis (not shown). Nonetheless, since tumor-infiltrating myeloid cells are thought to be suppressive in the setting of the tumor microenvironment (64), it will be worthwhile to further explore the potential suppressive properties of kidney-infiltrating myeloid cells in lupus and how this may in turn affect KIT phenotypes.

The metabolic profile of KITs may have implications for understanding and designing therapy for LN. Inhibition of mitochondrial and glucose signaling was recently shown to suppress disease in murine models of SLE (22). These studies hypothesized a role of metabolic pathways in attenuating peripheral $\mathrm{CD} 4^{+} \mathrm{T}$ cell activation. Based on the observations presented here, we suggest that, additionally, KITs - which are already metabolically suppressed - may be uniquely susceptible to further metabolic inhibition, which could cause further suppression or even apoptosis.

While, to our knowledge, exhaustion of $\mathrm{T}$ cells within target organs has not been linked to autoimmunity, a recent landmark study correlated the presence of exhausted CD8 ${ }^{+} \mathrm{T}$ cells in the peripheral blood with prolonged flare-free survival in lupus and antineutrophil cytoplasmic antibody (ANCA) vasculitis (65). Their presence in the blood of some patients who will progress more slowly may reflect a genetic proclivity in these patients for inducing exhaustion, which in turn could result in target-organ resistance to $\mathrm{T}$ cell-mediated damage. The origin of these prognostic cells, however, remains unknown. It could be assumed that circulating exhausted $\mathrm{CD} 8^{+} \mathrm{T}$ cells become exhausted in secondary lymphoid organs. Our data suggest an alternate scenario that connects exhaustion to lack of target-organ damage in nonprogressors: circulating exhausted $\mathrm{CD}^{+} \mathrm{T}$ cells could derive from cells that infiltrated organs, became exhausted at that site, and then recirculated. Regardless of which explanation applies, considering the 2 studies together raises the intriguing possibility that flares may be related to events that break homeostatic tolerance or exhaustion rather than de novo activation of new clones.

Our observations could also have implications for disease monitoring and novel therapy design as well as for therapies already in the clinic. With respect to disease stratification and tracking, an exhaustion phenotype in kidney biopsies might be predictive of disease outcomes or subtypes, analogous to blood signatures seen by McKinney et al. (65). Effective therapy could promote more rapid exhaustion in nephritis, for example, via PD-1 agonists. Furthermore, since exhausted $\mathrm{T}$ cells differ from both naive and activated $\mathrm{T}$ cells, it may be possible to design drugs that selectively eliminate such cells without unduly suppressing anti-foreign-activated T cells.

\section{Methods}

Animals. All mice were housed under specific pathogen-free conditions prior to use. MRL.Fas ${ }^{l p r}$ (MRL/lpr) (ages 18 to 24 weeks), Fcgr2b-/.Yaa (ages 26 to 32 weeks), and MRL.Tlr9 ${ }^{-/-}$(ages 9 to 10 months) mice were bred in our lab ( $\mathrm{Fcgr} 2 \mathrm{~b}^{-/-}$.Yaa mice were a gift from Silvia Bolland (NIAID, $\mathrm{NIH}$, Rockville, Maryland, USA), and $\operatorname{Tl} \mathrm{r}^{-/-}$mice (66) of a mixed genetic background (B6 and 129Sv) were bred to MRL/lpr mice in our colony and backcrossed for at least 10 generations. MRL/lpr, BALB/c, and B6 mice were purchased from the Jackson Laboratory. Lupus-prone mice were evaluated at the range of ages defined above when they met the additional criteria of being nephritic, as determined by a proteinuria level of 3 or 4 using colorimetric dipstick assay (Albustix; Bayer). In the experiments outlined in Supplemental Figures 1, 3, and 4, MRL/lpr mice were used at 11 to 12 weeks of age and B6 mice were used at 5 and 21 weeks of age, as specified in the figure legends.

Isolation of KITs. After sacrifice, spleens were removed and animals were perfused with $40 \mathrm{ml}$ HBSS until complete blanching of liver and kidney occurred. Kidneys were removed, and the capsular layer was dissected away. KITs were isolated using the Octodissociator (Miltenyi Biotech) in the presence of 1600 Kunitz units/ml collagenase D (Roche Diagnostics) and $0.2 \mathrm{mg} / \mathrm{ml}$ DNAse IV (Sigma-Aldrich) for 30 minutes at $37^{\circ} \mathrm{C}$. Digestion was quenched using metabolism buffer (2.5\% HEPES buffer [Gibco, Thermo Fisher Scientific], 0.5\% BSA, 2.5 mM EDTA pH 8.0 in PBS). Homogenates were allowed to settle for 15 minutes, and supernatant, which contained the majority of immune cells, was collected. RBC lysis was performed, and cells were filtered through a $70 \mu \mathrm{M}$ mesh. Splenocytes were isolated between 2 frosted glass slides using mechanical dissociation and filtered through a $70 \mu \mathrm{M}$ mesh filter after RBC lysis. Cells were then used in various assays of $\mathrm{T}$ cell function, as denoted in each experiment.

Flow cytometry. Single-cell suspensions were stained with antibodies against surface molecules in ice-cold PBS with 3\% calf serum and 5 mM EDTA in the presence of FcR-blocking Ab 2.4G2. Antibodies to the following targets were used: CD4 (clone GK1.5, BioLegend and in-house), CD8 (clone TIB-105, in-house), CD44 (clone Pgp-1, in-house), CD62L (clone Mel-14, BioLegend), TCR (clone H57-597, BioLegend), CD11b (clone M1/70, BioLegend), PD-1 (clone G4, in-house), Tim3 (clone 215008, R\&D), Lag3 (clone 4-10-C9, gift from Dario Vignali laboratory (University of Pittsburgh, Pittsburgh, Pennsylvania, USA), 2B4 (clone REA-524, Miltenyi Biotec), PD-L1 (clone 10F.9G2, BioLegend), and CD90.1 (clone 1A14, in house). Ghost BV510 (Tonbo) was used to exclude dead cells. For all in-house antibodies, hybridoma clones are commercially available. Antibodies were purified from HB101 (Irvine Scientific) or Hybridoma Serum Free Media (Gibco, Thermo Fisher Scientific) supernatants using protein G column (GE Healthcare) purification. Purity was monitored by acrylamide gel electrophoresis. Prior to conjugation, antibodies were concentrated and dialyzed into 0.1 M NaHCO3 pH 8.4 using Amicon 4 centrifugal device 30K MWCO Membrane (Millipore). For Alexa Fluor NHS esters (Thermo Fisher) and Pacific Blue (Thermo Fisher) conjugations, $10 \mu \mathrm{l}$ of a $10 \mathrm{mg} / \mathrm{ml}$ solution of dye in DMSO was added per $\mathrm{mg}$ of antibody at a $2 \mathrm{mg} / \mathrm{ml}$ concentration. The dye-antibody mixture was rotated in the dark for 1 hour at room temperature (RT). After an hour, the buffer was exchanged using Amicon 4 filter tubes to $1 \times \mathrm{PBS}$ with $0.05 \% \mathrm{NaN} 3$ and resuspended in a final concentration of $0.5 \mathrm{mg} / \mathrm{ml}$ in $1 \times \mathrm{PBS} / \mathrm{NaN} 3$. For biotin conjugation, $75 \mu \mathrm{l}$ of $1.5 \mathrm{mg} /$ $\mathrm{ml}$ Biotin XX, SSE (catalog B1606, Invitrogen) dissolved in DMSO was 
added per mg of antibody, which was at a $1 \mathrm{mg} / \mathrm{ml}$ concentration. The biotin-antibody mixture was rotated in the dark for 4 hours at RT, after which buffer was exchanged similarly to the way it was performed with Alexa Fluor dyes as described above. Appropriate dilutions and specificity were determined by titration on spleen cells. Cells were fixed in $1 \%$ paraformaldehyde in PBS. Samples analyzed for transcription factors were fixed/permeablized with the eBioscience FoxP3 staining kit. Antibodies to the following targets were used: Tcf1 (clone C63D9, Cell Signaling Technology) and Eomes (clone Dan11mag, eBioscience). All data were collected on an LSRII or Fortessa Analyzer (BD Bioscience) and analyzed with FlowJo software (Tree Star Inc.). An example of our generalized gating strategy can be seen in Supplemental Figure 10.

Intracellular cytokine assay. For intracytoplasmic cytokine staining, single-cell suspensions of cells $\left(5 \times 10^{6} /\right.$ well in $\left.200 \mu \mathrm{l}\right)$ were stimulated in vitro with $50 \mathrm{ng} / \mathrm{ml}$ PMA (EMD Millipore) and $1 \mu \mathrm{g}$ / $\mathrm{ml}$ ionomycin (EMD Millipore) for 4 hours in the presence of 1:1000 Brefeldin A (BioLegend). Cells were then harvested and stained with antibodies to CD8, CD4, TCR, and PD-1 before fixation. Intracellular staining was performed using Cytofix/Cytoperm and Perm/Wash buffers (BD). Permeabilized cells were then stained for IL-2 (JES65 H4, BioLegend), TNF- $\alpha$ (MP6-XT22, eBioscience), IFN- $\gamma$ (XMG-1.2, BioLegend), and IL-10 (JES5-16E3, BD Biosciences - Pharmingen) and analyzed by flow cytometry as described above.

Cell proliferation assay. Single-cell suspensions made from spleen or kidney were loaded with $10 \mu \mathrm{M}$ Cell Proliferation Dye eFluor 450 (eBioscience) according to the manufacturer's instructions (20 minutes at RT in PBS). They were then quenched on ice with RPMI 1640 supplemented with 5\% FCS (Gemini Benchmark), penicillin and streptomycin (Gibco, Thermo Fisher Scientific), 10 mM HEPES (Gibco, Thermo Fisher Scientific), and $2 \mathrm{mM}$ L-glutamine (Gibco, Thermo Fisher Scientific) for 5 minutes. Cells were stimulated in complete media with $1 \mu \mathrm{g} / \mathrm{ml}$ of plate-bound anti-CD3e (Tonbo) and 1 $\mu \mathrm{g} / \mathrm{ml}$ soluble anti-CD28 (Tonbo) in the presence of IL-2 (100 IU/ml) for indicated times. They were then collected and processed for flow cytometry analysis as described above.

Staining for flow cytometric analysis of metabolic status. Single-cell suspensions made from spleen or kidney were isolated in metabolism buffer. Samples were then incubated with $60 \mu \mathrm{M}$ 2NBDG (Cayman Chemical) in RPMI 1640 supplemented with 2\% FCS (Gemini Benchmark), penicillin and streptomycin (Gibco, Thermo Fisher Scientific), $10 \mathrm{mM}$ HEPES (Gibco, Thermo Fisher Scientific), and $2 \mathrm{mM}$ L-glutamine (Gibco, Thermo Fisher Scientific) for 30 minutes at $37^{\circ} \mathrm{C}$. Cells were surface stained and loaded with 20 nM MitoTracker FM (Thermo Fisher Scientific) or $10 \mathrm{nM}$ Mitostatus (BD Biosciences Pharmingen) dyes to measure mitochondrial mass and function and counterstained with antibodies, as indicated.

Seahorse analysis. Single-cell suspensions were made and stained as noted above for metabolism assays. Unfixed cells were sorted using FACSAria (BD Bioscience) for CD $4^{+} \mathrm{CD} 90.1^{+}$and $\mathrm{CD} 8^{+} \mathrm{CD} 90.1^{+}$populations. Cells were maintained on ice until they were plated on CellTak-coated Seahorse culture plates (200,000 T cells/well) and analyzed using Seahorse XFe96 (Agilent) as described (39).

RNA-seq analysis. T cells isolated from kidney and spleen were sorted using FACSAria (BD Bioscience). $1 \times 10^{6} \mathrm{CD}^{+}$and $\mathrm{CD} 8^{+} \mathrm{T}$ cells were isolated from matched kidneys and spleens from 3 individual MRL/lpr mice (age 23 weeks). RNA was isolated using the RNeasy Plus Micro Kit (QIAGEN). Samples were sequenced using
Illumina NextSeq 500 with 75 bp paired-end reads and aligned to the mm10 genome using the STAR aligner (67). The number of uniquely aligned reads ranged from 10 to 12 million. Gene-level counts were determined using featureCounts (42), and raw counts were analyzed for differential expression using the voom method (68) in the limma $\mathrm{R}$ package (43). All gene-set enrichments were preformed using the rankSumTestWithCorrelation function in limma, which explicitly corrects for correlation among genes in the gene set being interrogated. Specific gene sets used for GSEA correlation were derived as follows: for Figure 5, A and B, our gene set was correlated with genes defined in Supplemental Table 1 from ref. 44; for Figure 5C and Supplemental Figure 6, our gene set was correlated with genes defined in Supplemental Table 1 from ref. 36; for Supplemental Figure 7, our gene set was correlated with genes defined in Table 1 from ref. 37.

The presence of certain kidney-specific transcripts indicated unavoidable kidney cell contamination in the kidney sample; to prevent this from confounding our analysis, differential expression was performed only on $\mathrm{T}$ cell-expressed genes, which were defined as having at least an average of 20 counts in the splenic sample. All RNA-seq data were deposited in the NCBI's Gene Expression Omnibus database (GEO GSM3324012 and GSM3324023).

Immunofluorescence. Tissue sections were isolated from nephritic (age $>18$ weeks) MRL/lpr mice. Samples were fixed in 2\% PFA for 4 hours. Each sample was incubated in $30 \%$ sucrose phosphate buffer overnight at $4^{\circ} \mathrm{C}$ and then frozen in OCT compound (Tissue-Tek). Sections of $7 \mu \mathrm{m}$ were cut on a cryostat. Blocking was performed with $3 \%$ BSA and $10 \%$ rat serum in PBS for 30 minutes. Primary antibody staining was performed using anti-CD4-Al488 (clone GK1.5, in house), anti-CD8-Al647 (clone Lyt-2, in house), and anti-PD-L1-PE (clone 10F.9G2, BioLegend) antibodies. To improve photostability of PD-L1 staining, a secondary stain with anti-PE Al555 (Rockland) was performed. Sections were mounted using ProLong Gold Antifade Mountant with DAPI (Life Technologies). Once the mounting media cured, slides were sealed with nail polish. Imaging was performed on an Olympus IX83. Image display settings and exposure times were kept constant for all samples using CellSens software (Olympus).

LCMV infection. LCMV clone 13 virus was provided by Rafi Ahmed (Emory Vaccine Center, Atlanta, Georgia, USA) and propagated and titered as described previously (69). For chronic LCMVcl13 infection, mice were infected with $2 \times 10^{5}$ PFU i.v. Necropsy was on day 30 or later after infection.

Statistics. Statistical analysis was performed using Prism 6.0 (GraphPad). Paired 2-tailed Student's $t$ test or ANOVA analysis was performed where indicated. Each experiment shown is representative of at least 2 independent experiments unless otherwise indicated. $P<$ 0.05 was considered statistically significant, although lower $P$ values are indicated in individual figures.

Study approval. Animal studies were approved by the IACUC of the University of Pittsburgh.

\section{Author contributions}

JST and MJS conceived the project and designed experiments. JST performed most of the experiments and analyzed the data. LA performed proliferation assays and helped with flow staining. AVM performed Seahorse metabolism experiments. GMD assisted with design of metabolism and Seahorse experiments and data interpretation. MC and SS analyzed and interpreted the RNA seq data and 
preformed GSEA analysis. RAG and LPK assisted with data interpretation, experimental design, and paper edits. JST and MJS wrote the manuscript.

\section{Acknowledgments}

We would like to thank Dario Vignali for insights on $\mathrm{T}$ cell exhaustion and use of the Lag3 antibody; Kevin Nickerson for insights and comments on the manuscript; Andrea L. Workman for editorial comments; and Alex Rowe for useful discussions. This work was supported by NIH grants 2R01AR044077 and R01 AI137132 (to MJS) and the Lupus Insight Prize (to MJS). Additional support came from the Sidney-Kimmel Foundation for Cancer Research Scholar Award (SKF-015-039 to GMD), a Stand Up To Cancer - American Association of Cancer Research Innovative Research grant (SU2C-IRG-016-08 to GMD), an NIH Director's New Innovator Award (DP2AI136598 to GMD), and start-up funds to GMD through the Tumor Microenvironment Center at the University of Pittsburgh. JST was funded by NIH T32 grant 2T32AI0189443-06 and NIH Career Development Award 5KL2TR001856-02.

Address correspondence to: Mark J. Shlomchik, W1052 Biomedical Science Tower 200 Lothrop Street, Pittsburgh, Pennsylvania 15261, USA. Phone: 412.648.8771; Email: mshlomch@pitt.edu.
1. Davidson A. What is damaging the kidney in lupus nephritis? Nat Rev Rheumatol. 2016;12(3):143-153.

2. Hahn BH, et al. American College of Rheumatology guidelines for screening, treatment, and management of lupus nephritis. Arthritis Care Res (Hoboken). 2012;64(6):797-808.

3. Ioannidis JP, et al. Remission, relapse, and re-remission of proliferative lupus nephritis treated with cyclophosphamide. Kidney Int. 2000;57(1):258-264.

4. Jevnikar AM, Grusby MJ, Glimcher LH. Prevention of nephritis in major histocompatibility complex class II-deficient MRL-lpr mice. J Exp Med.1994;179(4):1137-1143.

5. Koh DR, Ho A, Rahemtulla A, Fung-Leung WP, Griesser H, Mak TW. Murine lupus in MRL/lpr mice lacking CD4 or CD8 T cells. Eur J Immunol. 1995;25(9):2558-2562.

6. Merino R, et al. Effect of long-term anti-CD4 or anti-CD8 treatment on the development of lpr CD4-CD8- double negative T cells and of the autoimmune syndrome in MRL-lpr/lpr mice. J Autoimmun. 1995;8(1):33-45.

7. Jabs DA, Burek CL, Hu Q, Kuppers RC, Lee B, Prendergast RA. Anti-CD4 monoclonal antibody therapy suppresses autoimmune disease in MRL/Mp-lpr/lpr mice. Cell Immunol. 1992;141(2):496-507.

8. Chan OT, Paliwal V, McNiff JM, Park SH, Bendelac A, Shlomchik MJ. Deficiency in beta(2)-microglobulin, but not CD1, accelerates spontaneous lupus skin disease while inhibiting nephritis in MRL-Fas(lpr) nice: an example of disease regulation at the organ level. JImmunol. 2001;167(5):2985-2990.

9. Christianson GJ, et al. beta2-microglobulin dependence of the lupus-like autoimmune syndrome of MRL-lpr mice. J Immunol. 1996;156(12):4932-4939.

10. Hannah J, Casian A, D'Cruz D. Tacrolimus use in lupus nephritis: A systematic review and meta-analysis. Autoimmun Rev. 2016;15(1):93-101.

11. Couzi L, et al. Predominance of CD8+ T lymphocytes among periglomerular infiltrating cells and link to the prognosis of class III and class IV lupus nephritis. Arthritis Rheum. 2007;56(7):2362-2370.

12. Hsieh C, Chang A, Brandt D, Guttikonda R, Utset TO, Clark MR. Predicting outcomes of lupus nephritis with tubulointerstitial inflammation and scarring. Arthritis Care Res (Hoboken). 2011;63(6):865-874.
13. Alexopoulos E, Seron D, Hartley RB, Cameron JS. Lupus nephritis: correlation of interstitial cells with glomerular function. Kidney Int. 1990;37(1):100-109.

14. Guo Z, Wang Y, Li R, Huang H, Wang R. Use of laser microdissection in the analysis of renalinfiltrating T cells in murine lupus. Cent Eur J Immunol. 2014;39(3):285-293.

15. Massengill SF, Goodenow MM, Sleasman JW. SLE nephritis is associated with an oligoclonal expansion of intrarenal T cells. Am J Kidney Dis. 1998;31(3):418-426.

16. Winchester R, et al. Immunologic characteristics of intrarenal T cells: trafficking of expanded CD8 $+\mathrm{T}$ cell $\beta$-chain clonotypes in progressive lupus nephritis. Arthritis Rheum. 2012;64(5):1589-1600.

17. Odegard JM, et al. ICOS-dependent extrafollicular helper T cells elicit IgG production via IL-21 in systemic autoimmunity. J Exp Med. 2008;205(12):2873-2886.

18. Wang Y, et al. Laser microdissection-based analysis of cytokine balance in the kidneys of patients with lupus nephritis. Clin Exp Immunol. 2010;159(1):1-10.

19. Crispín JC, et al. Expanded double negative $\mathrm{T}$ cells in patients with systemic lupus erythematosus produce IL-17 and infiltrate the kidneys. J Immunol. 2008;181(12):8761-8766.

20. Schmidt T, et al. Function of the Th17/interleukin17A immune response in murine lupus nephritis. Arthritis Rheumatol. 2015;67(2):475-487.

21. Moulton VR, Tsokos GC. Abnormalities of T cell signaling in systemic lupus erythematosus. Arthritis Res Ther. 2011;13(2):207.

22. Yin Y, et al. Normalization of CD $4+\mathrm{T}$ cell metabolism reverses lupus. Sci Transl Med. 2015;7(274):274ra18.

23. Alexander EL, Moyer C, Travlos GS, Roths JB, Murphy ED. Two histopathologic types of inflammatory vascular disease in MRL/Mp autoimmune mice. Model for human vasculitis in connective tissue disease. Arthritis Rheum. 1985;28(10):1146-1155.

24. Gu L, et al. Genetic determinants of autoimmune disease and coronary vasculitis in the MRL-lpr/ lpr mouse model of systemic lupus erythematosus. J Immunol. 1998;161(12):6999-7006.

25. Hang L, Theofilopoulos AN, Dixon FJ. A spontaneous rheumatoid arthritis-like disease in MRL/1 mice. J Exp Med.1982;155(6):1690-1701.

26. Berden JH, Hang L, McConahey PJ, Dixon FJ.
Analysis of vascular lesions in murine SLE. I. Association with serologic abnormalities. J Immunol. 1983;130(4):1699-1705.

27. Furukawa F, Tanaka H, Sekita K, Nakamura T, Horiguchi Y, Hamashima Y. Dermatopathological studies on skin lesions of MRL mice. Arch Dermatol Res. 1984;276(3):186-194.

28. Nickerson KM, et al. TLR9 regulates TLR7- and MyD88-dependent autoantibody production and disease in a murine model of lupus. J Immunol. 2010;184(4):1840-1848.

29. Woo J, Wright TM, Lemster B, Borochovitz D, Nalesnik MA, Thomson AW. Combined effects of FK506 (tacrolimus) and cyclophosphamide on atypical B220+ T cells, cytokine gene expression and disease activity in MRL/MpJ-lpr/lpr mice. Clin Exp Immunol. 1995;100(1):118-125.

30. Van Bruggen MC, Walgreen B, Rijke TP, Berden $\mathrm{JH}$. Attenuation of murine lupus nephritis by mycophenolate mofetil. JAm Soc Nephrol. 1998;9(8):1407-1415.

31. Shiraki M, Fujiwara M, Tomura S. Long term administration of cyclophosphamide in MRL/1 mice. I. The effects on the development of immunological abnormalities and lupus nephritis. Clin Exp Immunol. 1984;55(2):333-339.

32. Yamamoto K, Mori A, Nakahama T, Ito M, Okudaira H, Miyamoto T. Experimental treatment of autoimmune MRL-lpr/lpr mice with immunosuppressive compound FK506. Immunology. 1990;69(2):222-227.

33. Ahuja A, Shupe J, Dunn R, Kashgarian M, Kehry MR, Shlomchik MJ. Depletion of B cells in murine lupus: efficacy and resistance. JImmunol. 2007;179(5):3351-3361.

34. Perry D, Sang A, Yin Y, Zheng YY, Morel L. Murine models of systemic lupus erythematosus. J Biomed Biotechnol. 2011;2011:271694.

35. Wherry EJ, Kurachi M. Molecular and cellular insights into T cell exhaustion. Nat Rev Immunol. 2015;15(8):486-499.

36. Singer M, et al. A distinct gene module for dysfunction uncoupled from activation in tumorinfiltrating T cells. Cell. 2016;166(6):1500-1511.e9.

37. Wherry EJ, et al. Molecular signature of CD8+ $\mathrm{T}$ cell exhaustion during chronic viral infection. Immunity. 2007;27(4):670-684.

38. Anderson AC, Joller N, Kuchroo VK. Lag-3, Tim-3, and TIGIT: co-inhibitory receptors with specialized functions in immune regulation. Immunity. 2016;44(5):989-1004.

39. Scharping NE, et al. The tumor microenviron- 
ment represses $\mathrm{T}$ cell mitochondrial biogenesis to drive intratumoral $\mathrm{T}$ cell metabolic insufficiency and dysfunction. Immunity. 2016;45(3):701-703.

40. Bengsch B, et al. Bioenergetic insufficiencies due to metabolic alterations regulated by the inhibitory receptor PD-1 are an early driver of CD8(+) T cell exhaustion. Immunity. 2016;45(2):358-373.

41. Chang $\mathrm{CH}$, et al. Posttranscriptional control of $\mathrm{T}$ cell effector function by aerobic glycolysis. Cell. 2013;153(6):1239-1251.

42. Liao Y, Smyth GK, Shi W. featureCounts: an efficient general purpose program for assigning sequence reads to genomic features. Bioinformatics. 2014;30(7):923-930.

43. Ritchie ME, et al. limma powers differential expression analyses for RNA-sequencing and microarray studies. Nucleic Acids Res. 2015;43(7):e47.

44. Crawford A, et al. Molecular and transcriptional basis of $\mathrm{CD} 4^{+} \mathrm{T}$ cell dysfunction during chronic infection. Immunity. 2014;40(2):289-302.

45. Schwanhäusser B, et al. Corrigendum: Global quantification of mammalian gene expression control. Nature. 2013;495(7439):126-127.

46. Mazumder B, Li X, Barik S. Translation control: a multifaceted regulator of inflammatory response. JImmunol. 2010;184(7):3311-3319.

47. Villarino AV, et al. Posttranscriptional silencing of effector cytokine mRNA underlies the anergic phenotype of self-reactive T cells. Immunity. 2011;34(1):50-60.

48. Buggert $\mathrm{M}$, et al. T-bet and Eomes are differentially linked to the exhausted phenotype of CD8+ T cells in HIV infection. PLoS Pathog. 2014;10(7):e1004251.

49. Wu T, et al. The TCF1-Bcl6 axis counteracts type I interferon to repress exhaustion and maintain $\mathrm{T}$ cell stemness. Sci Immunol. 2016;1(6):eaai8593.

50. Ding H, et al. Delivering PD-1 inhibitory signal concomitant with blocking ICOS co-stimulation suppresses lupus-like syndrome in autoimmune BXSB mice. Clin Immunol. 2006;118(2-3):258-267.

51. Kasagi S, et al. Anti-programmed cell death 1 antibody reduces $\mathrm{CD} 4+\mathrm{PD}-1+\mathrm{T}$ cells and relieves the lupus-like nephritis of NZB/W F1 mice. J Immunol. 2010;184(5):2337-2347.

52. Lucas JA, Menke J, Rabacal WA, Schoen FJ, Sharpe AH, Kelley VR. Programmed death ligand 1 regulates a critical checkpoint for autoimmune myocarditis and pneumonitis in MRL mice. JImmunol. 2008;181(4):2513-2521.

53. Chen Y, et al. Expression of B7-H1 in inflammatory renal tubular epithelial cells. Nephron Exp Nephrol. 2006;102(3-4):e81-e92.

54. Bertsias GK, et al. Genetic, immunologic, and immunohistochemical analysis of the programmed death 1 /programmed death ligand 1 pathway in human systemic lupus erythematosus. Arthritis Rheum. 2009;60(1):207-218.

55. Pardoll DM. The blockade of immune checkpoints in cancer immunotherapy. Nat Rev Cancer. 2012;12(4):252-264.

56. Abdel-Wahab N, Shah M, Suarez-Almazor ME. Adverse events associated with immune checkpoint blockade in patients with cancer: a systematic review of case reports. PLOS ONE. 2016;11(7):e0160221.

57. Michot JM, et al. Immune-related adverse events with immune checkpoint blockade: a comprehensive review. Eur JCancer. 2016;54:139-148.

58. Ding H, Wu X, Gao W. PD-L1 is expressed by human renal tubular epithelial cells and suppresses T cell cytokine synthesis. Clin Immunol. 2005;115(2):184-191.
59. Starke A, et al. Renal tubular PD-L1 (CD274) suppresses alloreactive human T-cell responses. Kidney Int. 2010;78(1):38-47.

60. Lyford-Pike S, et al. Evidence for a role of the PD-1:PD-L1 pathway in immune resistance of HPV-associated head and neck squamous cell carcinoma. Cancer Res. 2013;73(6):1733-1741.

61. Paley MA, et al. Progenitor and terminal subsets of CD8+ T cells cooperate to contain chronic viral infection. Science. 2012;338(6111):1220-1225.

62. Schmitz JE, et al. Control of viremia in simian immunodeficiency virus infection by CD $8+$ lymphocytes. Science. 1999;283(5403):857-860.

63. Chen Y, Fry BC, Layton AT. Modeling glucose metabolism in the kidney. Bull Math Biol. 2016;78(6):1318-1336.

64. Corzo CA, et al. HIF-1 $\alpha$ regulates function and differentiation of myeloid-derived suppressor cells in the tumor microenvironment. J Exp Med. 2010;207(11):2439-2453.

65. McKinney EF, Lee JC, Jayne DR, Lyons PA, Smith KG. T-cell exhaustion, co-stimulation and clinical outcome in autoimmunity and infection. Nature. 2015;523(7562):612-616.

66. Hemmi H, et al. A Toll-like receptor recognizes bacterial DNA. Nature. 2000;408(6813):740-745.

67. Dobin A, et al. STAR: ultrafast universal RNA-seq aligner. Bioinformatics. 2013;29(1):15-21.

68. Law CW, Chen Y, Shi W, Smyth GK. voom: Precision weights unlock linear model analysis tools for RNA-seq read counts. Genome Biol. 2014;15(2):R29.

69. Ahmed R, Salmi A, Butler LD, Chiller JM, Oldstone MB. Selection of genetic variants of lymphocytic choriomeningitis virus in spleens of persistently infected mice. Role in suppression of cytotoxic T lymphocyte response and viral persistence. J Exp Med.1984;160(2):521-540. 